\title{
On Resistance and Inductance of Solid Conductors
}

\author{
Oldřich Coufal \\ Faculty of Electrical Engineering and Communication, Brno University of Technology, Technická 3082/12, 61600 Brno, Czech Republic
}

Correspondence should be addressed to Oldřich Coufal; coufal@feec.vutbr.cz

Received 18 December 2012; Accepted 13 March 2013

Academic Editor: Lucian Dascalescu

Copyright (C) 2013 Oldřich Coufal. This is an open access article distributed under the Creative Commons Attribution License, which permits unrestricted use, distribution, and reproduction in any medium, provided the original work is properly cited.

The concepts of resistance and inductance are strewn with misunderstanding and errors that result from inaccurate terminology, mistakes in their definitions, and from the fact that they are quantities that characterize elements of ideal electrical circuits as well as real solid conductors. This is shown unfavourably not only in the respective field of the theory of electrical engineering, but also in the calculation of resistance and inductance. In this paper, a brief theoretical analysis of the state of the art is given, and its results are applied in the calculation of the current density, resistance, and inductance of two coaxial solid tubular conductors.

\section{Introduction}

The fundamental concepts used in electrical engineering and their definitions are the subject of physics in the part electricity and magnetism [1]. Resistance $R$ and inductance $L$ are technical terms, and their meaning in electrical engineering should be strictly defined. In the basic circuit theory (CT), it is assumed that the values of $R$ and $L$ are given. The calculation of these quantities is the task of electromagnetic field theory (EM) [1]. EM and CT undoubtedly have much in common but the physical and mathematical characteristics of the basic quantities differ.

The basic quantities in Maxwell's theory of electromagnetism are the field vectors E, B, and J [2]. Electromagnetic phenomena in a circuit are expressed only by means of voltage across the elements and by currents flowing through them. If CT is used, the real electrical device is substituted by several ideal elements. In the following, instead of saying that a device is substituted by a resistor with resistance $R$ or by an inductor with inductance $L$, it will for brevity be said that a device is substituted by resistance $R$ or by inductance $L$.

In circuits with lumped parameters, voltages and currents may depend on time $t$ and are thus functions of $t$ but they are not functions of position. In circuits that satisfy this condition, the phenomena are referred to as quasi-stationary $[1,3]$ and the respective quantities are referred to as slowly varying [4]. Displacement current is usually neglected when examining these phenomena. In the present paper, quasistationary phenomena alone will be examined.

The concepts of resistance and inductance are coupled with misunderstanding and errors that result from inaccurate terminology, disregarding valid norms, from mistakes in their definitions and from the fact that they are quantities that characterize elements of ideal electrical circuits as well as real solid conductors. In the paper, a theoretical analysis of the state of the art is given, and its results are applied in the calculation of the resistance and inductance of two coaxial solid tubular conductors. These conductors are the simplest possible arrangement of conductors, except for the solitary conductor, which was examined already in [5]. The formulae for calculating $R$ and $L$ as derived by Thomson in [5] are not correct, inclusive of the frequency dependence of resistance; the solitary conductor is not a suitable model for the calculation of $L$, as has been proved in [6].

\section{Ohm's Law and Resistance}

2.1. Ohm's Law, Resistance, and Impedance in CT. By Ohm's law, the voltage between resistor poles is in proportion to the current flowing through the resistor:

$$
U=I R \text {. }
$$


Using the reciprocal value $1 / R=G$, Ohm's law can be written as

$$
I=G U
$$

In the present paper, the symbols $U$ or $U(t)$ and $I$ or $I(t)$ will denote voltage and current, respectively, and not the effective value of these quantities. Underlined symbols will denote complex numbers in the following.

In [7], the resistive two-terminal element is defined as passive two-terminal element characterized by a functional relation between the voltage across the terminals and the electric current in the element. The electric energy absorbed by a resistive two-terminal element is not recoverable as electric energy at the terminals. The resistive two-terminal element is characterized by a quantity denoted by $R$. The linear resistive two-terminal element is an ideal resistor (see [7]), and $R$ satisfies relation (1). The terms resistance (1) and resistance (2) (both denoted by the symbol $R$ ) and impedance are defined in [7]. In the following text, the denotations resistance- 1 and resistance- 2 are used to prevent mistaking them for relations (1) and (2); the word resistance refers comprehensively to the two resistances.

Definition 1. Resistance-1 for a resistive two-terminal element or two-terminal circuit with terminals $\mathrm{A}$ and $\mathrm{B}$ equals the voltage $U_{\mathrm{AB}}$ between the terminals divided by the electric current $I$ in the element or circuit:

$$
R=\frac{U_{\mathrm{AB}}(t)}{I(t)}, \quad R \geq 0,
$$

where the electric current is taken as positive if its direction is from A to B and negative in the opposite case (see [7]).

Definition 2 (see [7]). Impedance of passive linear twoterminal element or two-terminal circuit under sinusoidal conditions equals the quotient of the phasor $\underline{U}$ representing the voltage between the terminals divided by the phasor $\underline{I}$ representing the electric current in the element or circuit:

$$
\underline{Z}=\frac{\underline{U}}{\underline{I}} .
$$

Definition 3 (see [7]). Resistance-2 is the real part of an impedance $\underline{Z}$ :

$$
R=\Re(\underline{Z}) .
$$

In the current spoken and written utterances in electrical engineering, the term resistance is used without any further differentiation or specification. This leads not only to vague formulations, but also to erroneous statements. The terms defined in [7] also include conductance-1 and conductance-2, both of which are denoted by the symbol $G$. Conductance-1 equals the reciprocal value of resistance- 1 while conductance2 equals the real part of admittance.

2.2. Ohm's Law in EM. Ohm's law (1) is a consequence of the general relation [2]

$$
\mathbf{J}=\mathbf{J}(\mathbf{E}, \mathbf{B})
$$

The dependence of $\mathbf{J}$ on $\mathbf{B}$ is manifested markedly in an ionized gaseous medium (Hall effect). Throughout a remarkably wide range of conditions, in both solids and weakly ionized solutions, relation (6) proves to be linear [8]. The proportionality constant only depends on the material of the conductor but not on its shape:

$$
\mathbf{J}=\gamma \mathbf{E}
$$

In isotropic conductors, the conductivity $\gamma$ is a scalar and is equal to the reciprocal value of resistivity $(\gamma=1 / \varrho)$. In anisotropic conductors, $\gamma$ is a tensor.

Ohm's law (7) is remarkable in that it holds very accurately in many conductors and over a wide range of values of $E=$ $|\mathbf{E}|$. The validity of (7) can thus be assumed in the case that the changes in the field $\mathbf{E}$ or $\mathbf{J}$ are negligible over a time interval of $\approx 10^{-14} \mathrm{~s}$ in metallic conductors $[4,9]$. For a periodically changing field $\mathbf{E}$ with frequency $f$, law (7) holds if $f \ll$ $10^{14} \mathrm{~Hz}$.

2.3. Calculation of Resistance-1. It follows from the above that law (7) is proved on the assumption of the fulfilment of the respective conditions. To calculate resistance- 1 means to calculate $R$ in relation (1) or $G$ in relation (2). When deriving relation (7), the field $\mathbf{E}$ was assumed to be homogeneous in the conductor. By (7), this means that the field $\mathbf{J}$ is also homogeneous in the conductor. Any conductor placed in a nonconducting medium is a tube of the field $\mathbf{J}$ and by (7) also of the field E. Assume that the conductor is of length $l$, and its ends are equipotential surfaces. They are areas of constant potential, whose gradient is the field $\mathbf{E}$. Consider a part of this conductor, which is homogeneous (with $\gamma$ constant) and has constant fields $\mathbf{E}$ and $\mathbf{J}$, and this part is a current tube of crosssection $\Delta S$ between two equipotential levels, whose distance is $\Delta l$. On these assumptions, the vector $\Delta \mathbf{S}$ is parallel to $\Delta \mathbf{l}, \mathbf{E}$, and J. After multiplying (7) by the product $\Delta \mathbf{l} \cdot \Delta \mathbf{S}$ and after rewriting, we have

$$
\Delta U=\Delta R \Delta I
$$

where

$$
\Delta U=\mathbf{E} \cdot \Delta \mathbf{l}, \quad \Delta I=\mathbf{J} \cdot \Delta \mathbf{S}, \quad \Delta R=\frac{\varrho \Delta l}{\Delta S} .
$$

If the assumptions made for a part of the conductor hold for the whole conductor, then the conductor has a constant crosssection $S$. Equations that hold for the conductor are (8) and (9) but with $\Delta$ left out. Equation (8) is then law (1).

In the real circuit, the real conductor under consideration is replaced by a resistor which has resistance-1 $R$. Several resistances- $1 R_{i}, i=1,2, \ldots, n, n>1$, which satisfy relations (1) and (2), can be substituted by one resistor whose resistance- 1 is $R$. On the assumption of the validity of both Kirchhoff's laws, it holds for resistors connected in series or in parallel:

$$
\sum_{i=1}^{n} R_{i}=R
$$


or

$$
\sum_{i=1}^{n} G_{i}=G, \quad G_{i}=\frac{1}{R_{i}}, \quad G=\frac{1}{R} .
$$

When proving law (1) in the first paragraph of this section, it was assumed, among other things, that $\gamma$ and $\mathbf{J}$ are constant in the conductor. In the following we will prove the validity of law (1) also for the case when $\gamma=\gamma(P)$ and $\mathbf{J}=\mathbf{J}(P)$, where $P$ denotes a point in the conductor denoted by the symbol $V$. We will assume that the cross section of the conductor changes along conductor continuously and that the conductivity $\gamma$ in the conductor is a continuous and bounded function. These assumptions do not imply any loss of generality because a real conductor is made up of a finite number of such conductors and thus its resistance- 1 can be determined using relations (10) and (11).

The conductor is a tube of the field $\mathbf{J}$, and its ends are equipotential areas. The magnitudes of the cross sections at the conductor ends are $S_{b}$ and $S_{e}$. We will divide the area $S_{b}$ into $S_{i}, i=1,2, \ldots, n$. The sets $S_{i}$ are simply connected sets (see [10]) and every two of them have at most the boundary points in common. It holds that

$$
S_{b}=\bigcup_{i=1}^{n} S_{i}, \quad V=\bigcup_{i=1}^{n} T_{i}
$$

where $T_{i}$ is the tube of field $\mathbf{J}$ given by set $S_{i}$. By definition, connected sets $S_{i}$ are open sets. This means that they do not include their boundaries, and, consequently, the right and the left sides of relations (12) differ from each other by the set of measure zero, which is made up of the boundaries of the sets $S_{i}$ and $T_{i}$. In view of the continuity of $\gamma$, the current flowing through the boundaries is also zero. Inside every part of $S_{i}$ we choose arbitrarily a point $P_{i 0}$. We denote by $l_{i}$ the line of vector $\mathbf{J}$ which passes through the point $P_{i 0}$. We choose an integer $m>0$ and denote by $P_{i m}$ the point at which the line $l_{i}$ intersects the area $S_{e}$. Using the points $P_{i j}, j=1,2, \ldots, m$, we divide the part of each line $l_{i}, i=1,2, \ldots, n$, between the points $P_{i 0}$ and $P_{i m}$ into $m$ arcs of the same length $\Delta l$. Between every two points $P_{i, j-1}$ and $P_{i j}$, we choose arbitrarily a point $\bar{P}_{i j}$, that is, a total of $n \times m$ points. The equipotential area passing through the point $\bar{P}_{i j}$ intersects the tube $T_{i}$ in the area $S_{i j}$. We denote, respectively, $\mathbf{J}_{i j}$ and $\varrho_{i j}$, the current density and resistivity at the point $\bar{P}_{i j}$. The conductor $V$ can be substituted by conductors $V_{i j}, i=1,2, \ldots, n, j=1,2, \ldots, m$, with each conductor $V_{i j}$ having a constant cross-section $S_{i j}$, length $\Delta l$, constant resistivity $\varrho_{i j}$, and constant current density $\mathbf{J}_{i j}$. The conductors $V_{i j}$, where $i$ is constant and $j=1,2, \ldots, m$, are arranged in series and form a partial conductor $\bar{V}_{i}$. The conductors $\bar{V}_{i}, i=1,2, \ldots, n$, are connected in parallel. The resistance-1 of the conductor $\bar{V}_{i}$ depends on $m$ and $n$ and is denoted by $\bar{R}_{i}(m, n)$. By (9) and (10),

$$
\bar{R}_{i}(m, n)=\sum_{j=1}^{m} \frac{\varrho_{i j} \Delta l}{S_{i j}} .
$$

By (11), the conductance-1 of the conductor composed of all the parts $V_{i j}$ is

$$
\bar{G}(m, n)=\sum_{i=1}^{n} \frac{1}{\bar{R}_{i}(m, n)} .
$$

Formulae (13) and (14) express the physical essence of the calculation of resistance-1 or conductance- 1 and enable calculating numerically their approximate values. The conductor has been replaced by a series and parallel connection of conductors that are so small that their cross section can be considered constant, the same as their conductivity. The calculation of the exact values of resistance-1 and conductance-1 depends on the conductor shape and on the functions $\gamma(P)$ and $\mathbf{J}(P)$. The procedure of deriving the formula for the calculation of resistance- 1 and conductance- 1 in the general case is given in the following.

On the equipotential area $S_{b}$ of the conductor considered we choose an area $\Delta S_{b}$, which is a simply connected set. All the lines of vector J passing through the surface $\Delta S_{b}$ intersect the surface $S_{e}$ in a set $\Delta S_{e}$ and form a current tube. The part of this tube between the surfaces $\Delta S_{b}$ and $\Delta S_{e}$ is a conductor that will be denoted by $\Delta T$. Inside of $\Delta S_{b}$ we choose a point $P_{b}$. The line of vector $\mathbf{J}$ passing through this point will be denoted by $l$. This line intersects the surface $\Delta S_{e}$ at the point $P_{e}$. The length of line $l$ between the points $P_{b}$ and $P_{e}$, will be denoted $\ell$. A parameter $p$ will be introduced on the line $l$ such that to every point $P \in l$ lying between the points $P_{b}$ and $P_{e}$ the value of the parameter $p(P)$ is assigned, which is equal to the length of the part $l$ between the points $P_{b}$ and $P$. Thus, it holds that $p\left(P_{b}\right)=$ $0, p\left(P_{e}\right)=\ell$. Similarly, every equipotential surface $S \subset \Delta T$ can be assigned the value of the parameter $p(S)$, which equals the value of the parameter assigned to the intersection of the surface $S$ and the line $l$. The equipotential surfaces in $\Delta T$ do not intersect one another and are perpendicular to $l$. This can be exploited for choosing the coordinates of an arbitrary point $P \in \Delta T$. This is because the point $P$ can uniquely be assigned the equipotential surface $S$, on which the point $P$ lies, and the value of parameter $p(S)$. Two coordinates are sufficient to determine the position of $P$ on $S$. The two coordinates are not important, provided that the resistance- $1 \Delta R$ of the tube $\Delta T$ is determined on the assumption that $\gamma$ and $\mathbf{J}$ are constant on $S$ and $\gamma=\gamma(p), \quad \mathbf{J}=\mathbf{J}(p)$. Such a case was solved in [11] in a formally different way than in this paper. It follows from the above that for given $\gamma(p)$ and $\mathbf{J}(p)$, the surface $S$ only depends on $\Delta S_{b}$ and $p, S=S(p)$. There is a function $A(p)$ such that for every $S$ (more exactly for the magnitude of every surface $S$ ), it holds that $S(p)=\Delta S_{b} A(p)$, and in agreement with (10),

$$
\Delta R=\int_{0}^{\ell} \frac{\mathrm{d} p}{S(p) \gamma(p)}=\frac{1}{\Delta S_{b}} \int_{0}^{\ell} \frac{\mathrm{d} p}{A(p) \gamma(p)} .
$$

This formula can be rewritten using the generalized mean value theorem of integral calculus [12]

$$
\Delta R=\frac{1}{\Delta S_{b} A(\bar{p}) \gamma(\bar{p})} \int_{0}^{\ell} \mathrm{d} p=\frac{\ell}{\Delta S_{b} A(\bar{p}) \gamma(\bar{p})}, \quad \bar{p} \in[0, \ell] .
$$


Let us denote $\bar{A}=A(\bar{p}), \bar{\gamma}=\gamma(\bar{p})$. Relation (16) enables determining the conductance-1 $\Delta G$ of conductor $\Delta T$ :

$$
\Delta G=\frac{1}{\Delta R}=\frac{\Delta S_{b} \bar{A} \bar{\gamma}}{\ell} .
$$

If the surface $\Delta S_{b}$ contracts towards the point $P_{b}$ such that its diameter (the maximum distance of two points in $\Delta S_{b}$ ) converges to zero, then $\Delta S_{b} \rightarrow \mathrm{d} S_{b}$. The position of infinitesimal surface $\mathrm{d} S_{b}$ is given by the point $P_{b}$, that is, by its coordinates. The quantities $\ell, \bar{\gamma}$, and $\bar{A}$, that is, $\ell=\ell\left(P_{b}\right)$, $\bar{\gamma}=\bar{\gamma}\left(P_{b}\right)$, and $\bar{A}=\bar{A}\left(P_{b}\right)$, depend on $P_{b}$. For $\Delta S_{b} \rightarrow \mathrm{d} S_{b}$, $\Delta G$ converges to $\mathrm{d} G$. It follows from (17) that

$$
\mathrm{d} G=\frac{\bar{\gamma}\left(P_{b}\right) \bar{A}\left(P_{b}\right)}{\ell\left(P_{b}\right)} \mathrm{d} S_{b} .
$$

The formula for the calculation of the conductance-1 $G$ of the initially considered conductor, denoted above by $V$, is obtained by integrating the preceding relation:

$$
G=\int_{S_{b}} \frac{\bar{\gamma}\left(P_{b}\right) \bar{A}\left(P_{b}\right)}{\ell\left(P_{b}\right)} \mathrm{d} S_{b}
$$

\section{Faraday's Law and Inductance}

3.1. Current Filaments. In theoretical models, the complex motion of electric charges constituting current in a conductor is approximated either by the current characterized by the current density vector $\mathbf{J}$ or by a system of current filaments or by surface current. In the following, the word curve refers to a simple finite piecewise smooth closed curve [13].

The term current filament denotes two quantitatively and qualitatively different objects. The current filament- 1 is a curve in which current $I(t)$ of finite magnitude flows. Current filament- 1 is the idealization of a "thin" conductor whose cross section is small, relative to its length. The current density in filament-1 is infinitely large.

The current filament- 2 is used in the description of solid conductors. If the cross section of solid conductor is in the plane of coordinates $x y$ and the current density component $J_{z}$ at the point $(x, y)$ of the cross section is not identically equal to zero, then the current filament-2 passing through the point $(x, y)$ is formed by the lines of vector $\mathbf{J}$, which pass through the infinitesimal surface $\mathrm{d} x \mathrm{~d} y$. The infinitesimal current passing through this filament-2 is $\mathrm{d} I(t)=J_{z}(x, y, t) \mathrm{d} x \mathrm{~d} y$.

3.2. Magnetic Field of Current Filament-1. The magnetic field of filament-1, which is in the shape of a curve $C$, can be established using the Biot-Savart law:

$$
\mathbf{B}(x, y, z, t)=\frac{\mu_{0}}{4 \pi} I \int_{C} \frac{\mathrm{d} \mathbf{C} \times \mathbf{r}}{r^{3}},
$$

where $\mathbf{r}$ is the position vector of the point $(x, y, z)$, relative to the point at which the current element $I \mathrm{~d} \mathbf{C}$ occurs, $I=$ $I(t), t \in[0, \infty)$. The curve $C$ is rectifiable and can thus be approximated by a broken line with arbitrary precision. The broken line, denoted by $C_{\text {lin }}$ below, is formed by a sequence of line segments. The magnetic field produced by the current filament-1, which is in the shape of broken line $C_{\text {lin }}$, approximates the field produced by a current filament1 which is in the shape of curve $C$. The field of a filament1 in the shape of line $C_{\text {lin }}$ is a superposition of fields from the individual line segments that form $C_{\text {lin }}$. The formula for the calculation of the field of an arbitrary line segment of the current filament-1, which can be denoted $\mathscr{A}$, can be derived using (20).

The unit vector colinear with a vector a will be denoted by the superscript 1 on the right; that is, $\mathbf{a}^{1}=\mathbf{a} / a$. The line segment $\mathscr{A}$ has an initial point $\left(x_{0}, y_{0}, z_{0}\right)$ and an end point $\left(x_{1}, y_{1}, z_{1}\right)$. Let us further denote

$$
\begin{gathered}
\mathbf{a}=\left(x_{1}-x_{0}, y_{1}-y_{0}, z_{1}-z_{0}\right), \\
\mathbf{r}_{0}=\left(x-x_{0}, y-y_{0}, z-z_{0}\right), \\
\mathbf{r}_{1}=\left(x-x_{1}, y-y_{1}, z-z_{1}\right), \\
v=\mathbf{r}_{0} \cdot \mathbf{a}^{1} \\
\mathbf{d}=\mathbf{r}_{0}-v \mathbf{a}^{1}
\end{gathered}
$$

The line segment $\mathscr{A}$ is formed by the points $\left(C_{x}, C_{y}, C_{z}\right)$,

$$
\begin{aligned}
& C_{x}=x_{0}+p\left(x_{1}-x_{0}\right), \\
& C_{y}=y_{0}+p\left(y_{1}-y_{0}\right), \\
& C_{z}=z_{0}+p\left(z_{1}-z_{0}\right),
\end{aligned}
$$

where the parameter $p \in[0,1)$. The field produced by the line segment $\mathscr{A}$, through which the current $I$ flows from the initial point to the end point, is, by (20),

$$
\mathbf{B}_{\mathscr{A}}(x, y, z, t)=\frac{\mu_{0}}{4 \pi} I(\mathbf{a} \times \mathbf{d}) \int_{0}^{1} \frac{\mathrm{d} p}{\left(p^{2} a^{2}-2 p a v+r_{0}^{2}\right)^{3 / 2}}
$$

since $\mathrm{d} \mathbf{C} \times \mathbf{r}=\mathbf{a} \times \mathbf{d d} p$. After integrating and rewriting, we obtain

$$
\mathbf{B}_{\mathscr{A}}(x, y, z, t)=\frac{\mu_{0}}{4 \pi}\left(\mathbf{a}^{1} \times \mathbf{d}^{1}\right) \frac{I}{d}\left(\frac{v}{r_{0}}+\frac{a-v}{r_{1}}\right) .
$$

The vector $\mathbf{d}$ has its end at the point $(x, y, z)$ while its origin is the foot of the perpendicular dropped from the point $(x, y, z)$ onto the line $P$, on which the line segment $\mathscr{A}$ lies. $d$ is thus the distance of the point $(x, y, z)$ from the line $P$. Therefore, the expression on the right-hand side of relation (24) is not defined if $I \neq 0$ and the point $(x, y, z)$ is on the line $P$. For $(x, y, z) \in P$, the integral in (23) is improper, and therefore

$$
\mathbf{B}_{\mathscr{A}}(x, y, z, t)=\lim _{n \rightarrow \infty} \mathbf{B}_{\mathscr{A}}\left(x_{n}, y_{n}, z_{n}, t\right),
$$

where $\left\{\left(x_{n}, y_{n}, z_{n}\right)\right\}, n>1$, is an arbitrary sequence of points $\left(x_{n}, y_{n}, z_{n}\right) \notin P$, which for $n \rightarrow \infty$ converges to $(x, y, z) . n$ greater than 1 is chosen so that the denotation of the sequence points should not collide with the denotation of the initial 
and end points of the line segment $\mathscr{A}$. The quantities $\mathbf{r}_{\mathbf{0}}, \mathbf{r}_{\mathbf{1}}, \mathbf{d}$, and $v$, defined for the point $(x, y, z)$, will for the sequence points depend on $n$, which in the following is expressed by the subscript $n$. Obviously it holds that

$$
\lim _{n \rightarrow \infty} d_{n}=0
$$

The vectors $\mathbf{a}^{\mathbf{1}}$ and $\mathbf{d}^{\mathbf{1}}$ are perpendicular to each other and therefore their vector product in relation (24) is a unit vector. The magnitude of the field $\mathbf{B}_{\mathscr{A}}$ is thus determined by the expression to the right of the vector product in relation (24).

Let the point $(x, y, z)$ be an internal point of the segment $\mathscr{A}$. From the validity of (26), it follows that there exists a subscript $N, N>1$, such that for all $n>N$, it holds that $0<v_{n}<a$, and hence

$$
\lim _{n \rightarrow \infty} \frac{I}{d_{n}}\left(\frac{v_{n}}{r_{0 n}}+\frac{a-v_{n}}{r_{1 n}}\right)=\infty .
$$

This relation also holds when the point $(x, y, z)$ is the initial or end point of the line segment $\mathscr{A}$ and all the points $\left(x_{n}, y_{n}, z_{n}\right)$ lie in a plane that is perpendicular to $\mathscr{A}$, and passes through the point $(x, y, z)$. If the point $(x, y, z) \in P$ lies to the left of the point $\left(x_{0}, y_{0}, z_{0}\right)$ (for the point to the right of $\left(x_{1}, y_{1}, z_{1}\right)$ the procedure is analogous), then there exists a subscript $N, N>1$, such that for all $n>N$, it holds that $v_{n}<0$, $v_{n}=-\left(r_{0 n}^{2}-d_{n}^{2}\right)^{1 / 2}, a-v_{n}=\left(r_{1 n}^{2}-d_{n}^{2}\right)^{1 / 2}$,

$$
\frac{I}{d_{n}}\left(\frac{v_{n}}{r_{0 n}}+\frac{a-v_{n}}{r_{1 n}}\right)=\frac{I}{d_{n}}\left(-\sqrt{1-\frac{d_{n}^{2}}{r_{0 n}^{2}}}+\sqrt{1-\frac{d_{n}^{2}}{r_{1 n}^{2}}}\right) .
$$

From the validity of (26), it follows that for sufficiently large $n$, it holds that $d_{n} \leq r_{0 n}, d_{n} \leq r_{1 n}$. The square roots in (28) can thus be substituted by their expansion into the Taylor series [13], in which all but the first two terms are neglected; that is,

$$
\sqrt{1-\frac{d_{n}^{2}}{r_{0 n}^{2}}} \approx 1-\frac{d_{n}^{2}}{2 r_{0 n}^{2}}, \quad \sqrt{1-\frac{d_{n}^{2}}{r_{1 n}^{2}}} \approx 1-\frac{d_{n}^{2}}{2 r_{1 n}^{2}},
$$

yielding

$$
\frac{I}{2}\left(\frac{d_{n}}{r_{0 n}^{2}}-\frac{d_{n}}{r_{1 n}^{2}}\right)
$$

For $d_{n} \rightarrow 0$, the limit of this expression equals zero, so that

$$
\lim _{n \rightarrow \infty} B_{\mathscr{A}}\left(x_{n}, y_{n}, z_{n}, t\right)=0
$$

and hence $B_{\mathscr{A}}(x, y, z, t)=0$.

The integral in (23), if improper, converges, and its value is finite if the value of the limit in expression (25) does not depend on the choice of the sequence $\left\{\left(x_{n}, y_{n}, z_{n}\right)\right\}$. For $(x, y, z)=\left(x_{0}, y_{0}, z_{0}\right)$ and for $(x, y, z)=\left(x_{1}, y_{1}, z_{1}\right)$, this integral does not converge because the value of the limit in expression (25) depends on the choice of the sequence $\left\{\left(x_{n}, y_{n}, z_{n}\right)\right\}$ and equals either $\infty$ or zero or the limit does not exist.
Up to now we have proved that the field $\mathbf{B}_{\mathscr{A}}$

(i) outside of $P$ is given by relation (24),

(ii) is not defined on the line segment $\mathscr{A}$, inclusive of its end points, and

(iii) is zero on the line $P$ outside $\mathscr{A}$.

Since the field of current filament-1, which is in the shape of curve $C$, can be approximated with arbitrary precision by the field of current filament- 1 in the shape of $C_{\text {lin }}$, it is proved that the field $\mathbf{B}$ is not defined on current filament-1.

3.3. Magnetic Field of Current Defined by Current Density. The current density $\mathbf{J}=\mathbf{J}(x, y, z, t)$ is defined on the Cartesian product $V \times[0, \infty)$, where $V$ is a closed set of points $(x, y, z)$ and $t \in[0, \infty)$. We assume that for $(x, y, z) \notin V$, there is $J=0$. The set $V$ is realized by conductors and insulants and it cannot be ruled out that at some of its points there is $J=0$. $V$ has the property that with each of its points it also contains the whole line of the vector $\mathbf{J}$ passing through this point, and thus the flux of vector $\mathbf{J}$ through the boundary of the set $V$ (the conductor surface) is zero. In view of the assumption that $V$ is a closed set, this means that the motion of electric charge across the conductor surface in the direction of a tangent to the conductor surface cannot be ruled out; at the same time, this is not a case of surface current (through a current layer). The magnetic field can be established using the Biot-Savart law:

$$
\mathbf{B}(x, y, z, t)=\frac{\mu_{0}}{4 \pi} \int_{V} \frac{\mathbf{J} \times \mathbf{r}}{r^{3}} \mathrm{~d} V,
$$

where $\mathbf{r}$ is the position vector of the point $(x, y, z)$, relative to the point at which there is the current element $\mathbf{J} \mathrm{d} V$. No existence of currents outside of $V$ is assumed, so that the definition range of density $\mathbf{J}$ can be extended to the whole space; outside of $V$ it holds that $J=0$. In reality (from the macroscopic viewpoint), the current density cannot be other than piecewise continuous and therefore it is bounded. According to [10], the field $\mathbf{B}$ given by relation (32) is thus continuous at all the points of the space. The essence of calculating the integral in (32) consists in substituting the conductors in the set $V$ by current filaments- 2 and then superposing the elementary fields produced by individual filaments.

3.4. Faraday's Law and Magnetic Flux. The term inductance is closely related to the induction law. Faraday's law of electromagnetic induction is expressed by Maxwell's equation in the integral form

$$
\mathscr{E}_{C}=\oint_{C} \mathbf{E} \cdot \mathrm{d} \mathbf{C}=-\frac{\mathrm{d} \Phi}{\mathrm{d} t},
$$

where $C$ is a closed curve, the integral of the vector of intensity of field $\mathbf{E}$ along $C$ is the electromotoric force $\mathscr{E}_{C}$, and $\Phi$ is the flux of the vector of magnetic field $\mathbf{B}$ (magnetic flux) through an arbitrary simple finite piecewise smooth surface $S$ [13], in the following only surface, bounded by the curve $C$ :

$$
\Phi=\int_{S} \mathbf{B} \cdot \mathrm{d} \mathbf{S} .
$$


It is further assumed that the position and shape of the curve $C$ do not depend on $t$. $\Phi$ does not depend on the shape of the surface $S$, which is easy to prove if we express B by means of the vector potential and apply Stokes' theorem [4]. Stokes' theorem can, however, be applied only if its assumptions are fulfilled. Similarly, relations (33) and (34) make sense only when the respective integrals and derivatives are available. This means, inter alia, that the field $\mathbf{B}$ cannot be arbitrary. If the field $\mathbf{B}$ is produced by a current given by the current density, then by Section 3.3, the field $\mathbf{B}$ is continuous and its magnitude $B$ is bounded at all points of the space, and therefore the integral on the right-hand side of relation (34) always exists and is finite. Every curve $C$ can thus be uniquely assigned a linked flux [14] $\Phi=\Phi(C)$. The curve $C$ is arbitrary (except for the above assumptions); its position and shape need in no way be linked with the conductors considered, that is, with $V$ or with the lines of the vector $\mathbf{J}$ [15].

It is often argued that magnetic flux is linked with the conductor, which is incorrect or inaccurate at best. In fact, there are infinitely many curves in a real solid conductor, having different linked fluxes. This ambiguity is removed by the assumption of a closed conductor which is a filament-1. It will be proved below that this is a fundamental error because the linked flux of filament-1 has not been defined.

Let then current filament-1 have the shape of a closed curve $C$ approximated by a broken line $C_{\text {lin }}$. The linked flux $\Phi\left(C_{\text {lin }}\right)$ is the flux $\Phi(S)$ of vector B through a surface $S$ bounded by a curve $C_{\text {lin }}$. We choose an arbitrary line segment $\mathscr{A} \subset C_{\text {lin }}$. The flux $\Phi$ does not depend on $S$, so that $S$ may comprise a rectangle $\mathscr{R}$, one side of which lies on $\mathscr{A}$. It holds that $\Phi(S)=\Phi(\mathscr{R})+\Phi(S-\mathscr{R})$. We will show that the value of $\Phi(\mathscr{R})$ is infinitely large, which will prove that the flux $\Phi(S)$, too, is of infinitely large value and hence not defined.

Without loss of generality, we choose the system of Cartesian coordinates $x y z$ such that the line segment $\mathscr{A}$ of length $a>0$ lies on the axis $z$; its initial point is the origin of the system of coordinates, and the rectangle $\mathscr{R}$ lies in the first quadrant of the plane $x z$. The flux $\Phi(\mathscr{R})$ is a sum of the fluxes of fields generated by line segments of the curve $C_{\text {lin }}$. The flux of the field produced by the line segment $\mathscr{A}$ will be denoted by $\Phi_{\mathscr{A}}(\mathscr{R})$. The field generated by the curve $C_{\text {lin }}$ can be calculated using relation (24). The field B produced by the segment line $\mathscr{A}$ in the rectangle $\mathscr{R}$ has only the $y$ component $B_{\mathscr{A} y}$ nonzero and is given by relation (24), where $\mathbf{a}=(0,0, a), \mathbf{r}_{0}=(x, 0, z), \mathbf{r}_{1}=(x, 0, z-a), v=z$, and $\mathbf{d}=(x, 0,0)$. The rectangle $\mathscr{R}$ is the Cartesian product of intervals $\left[x_{b}, x_{e}\right] \times[0, a]$, where $0=x_{b}<x_{e}$, and hence

$$
\Phi_{\mathscr{A}}(\mathscr{R})=\int_{x_{b}}^{x_{e}} \int_{0}^{a} B_{\mathscr{A} y}(x, 0, z, t) \mathrm{d} z \mathrm{~d} x .
$$

As assumed, $x_{b}=0$, so that the integral on the right-hand side of (35) is improper because the field $\mathbf{B}_{\mathscr{A}}$ is not defined on $\mathscr{A}$. We calculate the integral in (35) for $x_{b}>0$ and via a limit transition for $x_{b} \rightarrow 0+$, we determine $\Phi_{\mathscr{A}}(\mathscr{R})$ [13]. For $x_{b}>0$, it holds that

$$
\begin{aligned}
& \Phi_{\mathscr{A}}(\mathscr{R})=\frac{\mu_{0} I}{2 \pi}\left(a \ln \frac{a+\sqrt{x_{b}^{2}+a^{2}}}{x_{b}}+x_{b}-\sqrt{a^{2}+x_{b}^{2}}\right. \\
& \left.-a \ln \frac{a+\sqrt{x_{e}^{2}+a^{2}}}{x_{e}}-x_{e}+\sqrt{a^{2}+x_{e}^{2}}\right) .
\end{aligned}
$$

In view of the assumptions made, the limit of the first term on the right-hand side of (36) for $x_{b} \rightarrow 0+$ is equal to $+\infty$; the limit of the remaining terms has a finite value. This proves that the flux $\Phi_{\mathscr{A}}(\mathscr{R})$ is not defined. From this, it follows that the linked flux $\Phi(C)$ is not defined either unless $C$ is a filament- 1 .

3.5. On Inductance and Its Definition. According to [7], ideal inductor is defined in CT as a linear inductive two-terminal element. The electric energy absorbed by an inductive twoterminal element is stored in magnetic form and is completely restitutable. In [7], inductance is defined by the following definition.

Definition 4. Inductance for an inductive two-terminal element with terminals A and B is the quotient of the linked flux $\Psi$ between the terminals divided by the electric current $I$ in the element

$$
L=\frac{\Psi(t)}{I(t)}
$$

where the sign of the linked flux is determined by taking the voltage, in the time integral defining it, as the difference of the electric potentials at terminals $\mathrm{A}$ and $\mathrm{B}$ and where the current is taken as positive if its direction is from $\mathrm{A}$ to $\mathrm{B}$ and negative in the opposite case.

In $\mathrm{CT}$,

$$
\Psi(t)=\int_{t_{0}}^{t} U_{\mathrm{AB}}(\tau) \mathrm{d} \tau,
$$

where $t_{0}$ is any instant before the first supply of electric energy [7]. The linked flux determined by relation (38) is useful only in CT because an inductor can in CT be only associated with voltage and current. The ideal inductor is dimensionless and does not produce any concrete field B. Equality (37) can be rewritten as $\Psi(t)=L I(t)$ and differentiated with respect to $t$. If $L$ does not depend on $t$, then

$$
U_{\mathrm{AB}}(t)=L \frac{\mathrm{d} I(t)}{\mathrm{d} t}
$$

If $L$ depends on $t$, the differential inductance $L_{\mathrm{d}}=\mathrm{d} \Psi / \mathrm{d} I$ is defined [7], which is constant only over a small interval of current. From the viewpoint of the objectives of the present paper, it is not necessary to deal with differential 
inductance because it does not bring anything new as regards the methodology: $L_{\mathrm{d}}=L$ for an ideal inductor.

Prior to calculating the resistance and inductance, it is necessary to replace the real solid conductor by resistors and inductors. An equivalent schematic diagram of the real solid conductor needs to be constructed. In sinusoidal conditions [7], this means that the conductor is replaced by an appropriate impedance. Conductor inductance then becomes inductor inductance in the equivalent schematic diagram and is given by relation (39).

\section{Resistance and Inductance of Two Coaxial Tubular Conductors}

4.1. Description of Conductors. Consider a pair of coaxial solid tubular conductors. Conductor cross sections and their position in the system of Cartesian coordinates $x y z$ are evident in Figure 1. In addition to the Cartesian system, the system of cylindrical coordinates $r \phi z$ is also used. But because of the symmetry with respect to axis $z$, the $\phi$ coordinate will be to no avail. The cross section of the inner or of the outer conductor is determined by the inequalities

$$
r_{i 0} \leq r \leq r_{\text {in }} \text { or } r_{o n} \leq r \leq r_{o 0},
$$

respectively. The quantities relating to the inner or the outer conductors are in the following denoted with the subscript $i$ or $o$, respectively, while the subscript $c$ denotes either $i$ or $o$. The permeability of conductors and of the insulation between the conductors is assumed to be $\mu_{0}$. The two conductors have the direction of axis $z$ and are connected to an ideal voltage source. An electrical appliance is connected to their other end. The source voltage and current $I$ in the conductors depend only on time $t \in[0, \infty)$. Conductor resistivity is $\varrho_{c}$, and the ratio of conductor cross-section magnitudes is denoted by

$$
q=\frac{r_{o 0}^{2}-r_{o n}^{2}}{r_{i n}^{2}-r_{i 0}^{2}}
$$

In view of the symmetry of the arrangement of conductors, it is assumed that current density vector $\mathbf{J}$ is parallel to the axis $z$ and depends only on $r$ and $t . \mathbf{J}=\left(0,0, J_{c}\right)$, where the function $J_{c}=J_{c}(r, t)$ is defined for $r \in\left[r_{i 0}, r_{o 0}\right]$ and

$$
\begin{gathered}
J_{c}(r, t)=0, \quad \text { for } r \in\left(r_{i n}, r_{o n}\right), \\
J_{c}(r, t)=J_{i}(r, t), \quad \text { for } r \in\left[r_{i 0}, r_{i n}\right], \\
J_{c}(r, t)=J_{o}(r, t), \quad \text { for } r \in\left[r_{o n}, r_{o 0}\right] .
\end{gathered}
$$

The magnitude $B$ of the field vector $\mathbf{B}$ also depends on only $r$ and $t$. The fact that the current $I$ and vectors $\mathbf{J}, \mathbf{B}$ do not depend on $z$ actually means that an infinitely high propagation velocity of electromagnetic field is assumed. In such a case, the current is considered to be slowly varying [4].

4.2. Partial Loops. Let $D_{n}$ be a division of the interval $\left[r_{i 0}, r_{i n}\right]$, determined by the points $r_{i 0}<r_{i 1}<r_{i 2}<\cdots<r_{i n}$. The points

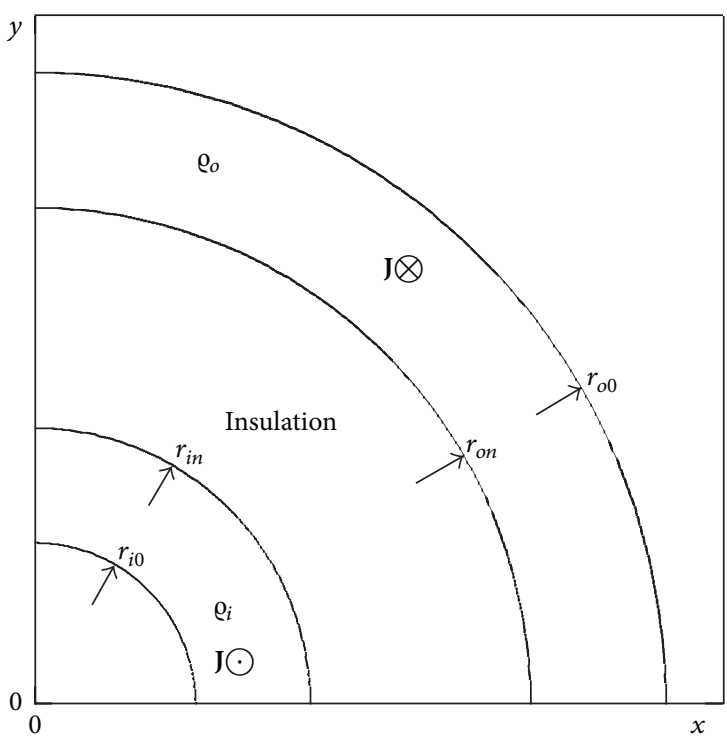

FIGURE 1: A quarter of the cross section of coaxial conductors and of the insulation between the conductors.

$r_{i 0}, r_{i 1}, \ldots, r_{i n}$ will be mapped via mapping $m$ onto the points $r_{o 0}>r_{o 1}>\cdots>r_{o n}$ in the interval $\left[r_{o n}, r_{o 0}\right]$; that is, the interval $\mathfrak{\Im}_{i k}=\left(r_{i, k-1}, r_{i k}\right]$ will be mapped onto the interval $\mathfrak{J}_{o k}=\left[r_{o k}, r_{o, k-1}\right)$. Mapping $m$ is a one-to-one mapping of the intervals $\left[r_{i 0}, r_{i n}\right]$ and $\left[r_{o n}, r_{o 0}\right]$. To determine $m$ means to determine the lines of vector $\mathbf{J}$ in the conductors, according to $[16]$

$$
m\left(r_{i}\right)=r_{o}=\sqrt{r_{o n}^{2}+q\left(r_{i n}^{2}-r_{i}^{2}\right)} .
$$

For simplicity it is assumed that $D_{n}$ is chosen such that for $k=1,2, \ldots, n$, and it holds that

$$
a=\pi\left(r_{i k}^{2}-r_{i, k-1}^{2}\right), \quad a=\frac{\pi\left(r_{i n}^{2}-r_{i 0}^{2}\right)}{n} .
$$

In consequence of relation (43), it holds for $k=1,2, \ldots, n$ that

$$
q a=\pi\left(r_{o, k-1}^{2}-r_{o k}^{2}\right) .
$$

Via division $D_{n}$, coaxial conductors can be divided into $n$ partial conductors, with the cross section of the $k$ th conductor being an annulus. The annulus of the $k$ th partial conductor in the inner conductor or in the outer conductor is determined by the radii $r_{i, k-1}, r_{i k}$ or $r_{o k}, r_{o, k-1}$. The $k$ th partial conductor in the inner conductor and the $k$ th partial conductor in the outer conductor form the $k$ th partial loop.

Conductor resistivity is constant, and so current density in the coaxial conductors will be continuous with respect to $r$ and can therefore be approximated with arbitrary accuracy by a function that is constant by parts. It can therefore be assumed that on the $k$ th partial interval $\mathfrak{I}_{i k}$, or $\mathfrak{I}_{o k}$, the current density magnitude $J_{i k}(t)$, or $J_{o k}(t)$, is independent of $r$. The current flowing through the $k$ th partial loop is $I_{k}(t)$, so that

$$
I_{k}(t)=a J_{i k}(t)=-q a J_{o k}(t)
$$


From relation (46) it follows that

$$
J_{o k}(t)=-\frac{J_{i k}(t)}{q} .
$$

The cross section of each of the conductors through the plane $z=$ const. is an equipotential area. In this paper, the segment of the two conductors between the planes $z=z_{1}$ and $z=z_{2}$, where $z_{1}<z_{2}, z_{2}-z_{1}=z_{21}$, will be considered. Across the two conductors in the plane $z=z_{1}$ or $z=z_{2}$ is a voltage $V\left(z_{1}, t\right)$ or $V\left(z_{2}, t\right)$.

A segment of the conductor between the planes $z=z_{1}$ and $z=z_{2}$ can be replaced by $n$ lumped parameter circuits. Current in the $k$ th partial loop depends on the resitance- 1 of the $k$ th partial loop. Resistances- 1 are given by the resistivities $\varrho_{i}, \varrho_{o}$ and the segment length $z_{21}$. The voltage induced in the segment of the $k$ th partial loop can be determined using the procedure described below.

Let us choose the points $c_{i 1}<c_{i 2}<\cdots<c_{i n}$ and $c_{o k}=$ $m\left(c_{i k}\right), k=1,2, \ldots, n$, such that $r_{i, k-1} \leq c_{i k} \leq r_{i k}$. Let us denote

$$
\Phi_{k}=\int_{A_{k}} \mathbf{B} \cdot \mathrm{d} \mathbf{A}_{k}
$$

with $A_{k}$ being a rectangle $\left[c_{i k}, c_{o k}\right] \times\left[z_{1}, z_{2}\right]$ in the plane $y=0$. According to Faraday's law, a voltage $\partial \Phi_{k} / \partial t$ is induced around the boundary of the rectangle $A_{k}$. If we take into consideration the fact that the magnetic field $\mathbf{B}$ produced by the current in the conductors under consideration has on the circle $r=$ const. the direction of the tangent to this circle, then

$$
\frac{\partial \Phi_{k}}{\partial t}=z_{21} \frac{\partial}{\partial t} \sum_{\ell=1}^{n} J_{i \ell}(t) \phi_{k \ell}
$$

where

$$
\phi_{k \ell}=\int_{c_{i k}}^{c_{o k}}\left[\mathscr{B}_{i \ell}(r)-\frac{\mathscr{B}_{o \ell}(r)}{q}\right] \mathrm{d} r .
$$

$\mathscr{B}_{i \ell}(r)$ or $\mathscr{B}_{o \ell}(r)$ is a magnetic field produced by the $\ell$ th partial conductor in the inner or the outer conductor per unit current density. For given $c_{c 1}, c_{c 2}, \ldots, c_{c n}$, the magnetic fluxes considered depend on $t$ alone. The function $J_{i \ell}(t)$ on the right-hand side of relation (49) also depends on $t$ alone, and therefore the derivative $\partial / \partial t$ can be replaced by the derivative $\mathrm{d} / \mathrm{d} t$ in (49). For a sufficiently large $n$ the voltage $U_{L k}$ induced in the segment of the $k$ th partial loop differs little from $\mathrm{d} \Phi_{k} / \mathrm{d} t$.

For the equivalent circuit of the segment of the $k$ th partial loop, it holds that

$$
U_{R i k}(t)+U_{R o k}(t)+U_{L k}(t)+V\left(z_{2}, t\right)-V\left(z_{1}, t\right)=0 .
$$

This equation, written for the segment of all partial loops, forms after modification a system of $n$ ordinary differential equations with constant coefficients with respect to the functions $J_{i k}, k=1,2 \ldots, n$,

$$
\left(\varrho_{i}+\frac{\varrho_{o}}{q}\right) J_{i k}(t)+\sum_{\ell=1}^{n} \phi_{k \ell} \frac{\mathrm{d} J_{i \ell}(t)}{\mathrm{d} t}=U(t), \quad k=1,2 \ldots, n,
$$

where $U(t)=\left[V\left(z_{1}, t\right)-V\left(z_{2}, t\right)\right] / z_{21}$.
In the steady state with a sinusoidal voltage source $U(t)=$ $\widehat{U} \sin (\omega t+\alpha)$, it is of advantage to solve the system (52) for the complex voltage $\underline{U} \exp (j \omega t)$ and for the current densities $J_{i k} \exp (j \omega t)$ on the partial intervals $\mathfrak{\Im}_{i k}, k=1,2, \ldots, n$, where $\underline{U}=\widehat{U} \exp (j \alpha), \underline{J}_{i k}=\widehat{J}_{i k} \exp \left(j \beta_{i k}\right)$, and thus $J_{i k}(t)=$ $\widehat{J}_{i k} \sin \left(\omega t+\beta_{i k}\right)$. Substituting phasors into (52) yields, after minor modification, a system of linear algebraic equations with complex coefficients for the unknown phasors $J_{i k}, k=$ $1,2, \ldots, n$,

$$
\left(\varrho_{i}+\frac{\varrho_{o}}{q}\right) \underline{J}_{i k}+j \omega \sum_{\ell=1}^{n} \phi_{k \ell} \underline{J}_{i \ell}=\underline{U}, \quad k=1,2 \ldots, n .
$$

The system (53) was solved using the Gaussian elimination with the selection of the maximum element in the matrix of the system. The maximum value of $n$ is given by the size of RAM. The calculations were carried out on a computer with 4 Gbyte RAM, which enables solving up to 10000 equations. The coefficients in the main diagonal of system (53) include the element $\varrho_{i}+\varrho_{o} / q$, whose value does not depend on $n$ while the value of the other coefficients $\phi_{k \ell}$ decreases with increasing $n$. Thus with increasing $n$, the main diagonal of the system matrix becomes dominant, which undoubtedly contributes to the stability of the solution of system (53). As regards the low values of the amplitude of densities $\widehat{J}_{i \ell}$, all that has been given about this problematic in $[16,17]$ holds.

The resistance- $1 R_{k}$ per $1 \mathrm{~m}$ of the considered segment of partial loops is for all partial loops identical because the validity of (44) is assumed. For the segment of the $k$ th loop, it holds that

$$
R_{k}=\frac{\varrho_{i}+\varrho_{o} q^{-1}}{a}, \quad k=1,2, \ldots, n
$$

The solution of (53) for specific cases is given in [16]. But attention should be drawn to an error. In Figures 4 and 6 in [16] incorrect values of the initial phase $\beta_{o k}$ are given (the denotation used in [16], is $\alpha_{o k}$ ) in the partial conductors of the outer coaxial conductor. In the figures mentioned, the validity of relation (47) was ignored, so that $180^{\circ}$ needs to be added to the values $\alpha_{o k}$.

4.3. Current Density in Conductors. The calculation of current density in a pair of coaxial tubular conductors and the determination of its resistance and inductance will be performed via the solution of equation system (53) in the case that the conductors are supplied from a source of sinusoidal voltage.

All the calculation results given below were obtained for

$$
c_{i k}=r_{i, k-1}+\frac{k-1}{n}\left(r_{i k}-r_{i, k-1}\right), \quad k=1,2, \ldots, n .
$$

The current density resulting from the solution of equation system (53) can be considered as superposition of two current densities. The first density is generated by the voltage source; the second density is the density of induced current. The first current density is determined by the phasors $J_{-c k}^{\text {sou }}$ and the second by the phasors $\underline{J}_{c k}^{\text {ind }}, c=i, o ; k=1,2, \ldots, n$. The 


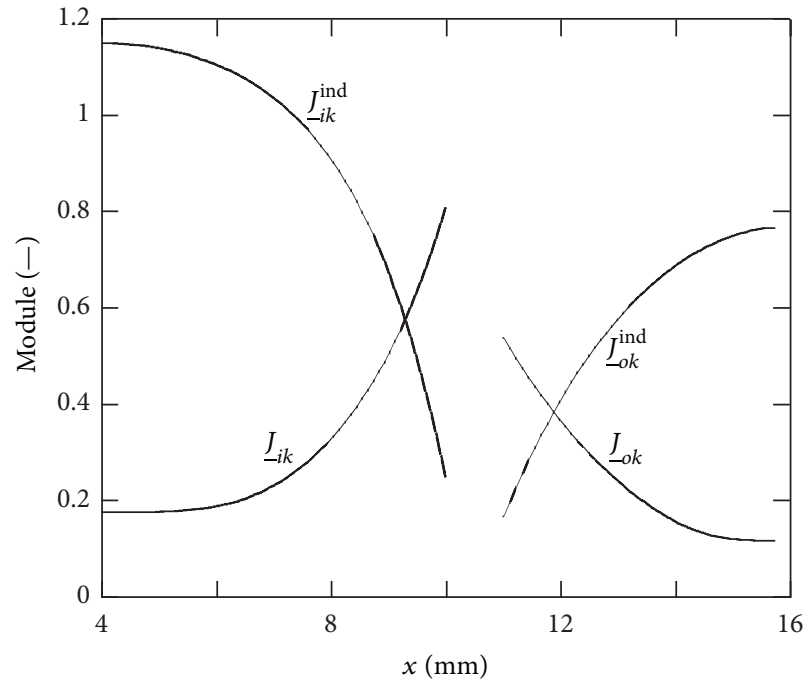

FIGURE 2: Dependence of phasor module of resultant current density $J_{c k}$ and of phasor module of induced current density $J_{-c k}^{\text {ind }}$ on distance from axis of conductors in Example 1 for $f=10^{3} \mathrm{~Hz}, n=1000$. The module values have been normalized with respect to $\widehat{J}_{i k}^{\text {sou }}=2.842 \times$ $10^{7} \mathrm{~A} \cdot \mathrm{m}^{-2}$.

partial conductors have identically large cross sections and therefore $J_{-c k}^{\text {sou }}$ do not depend on $k$,

$$
J_{i k}^{\text {sou }}=\frac{\underline{U}}{\varrho_{i}+\varrho_{o} q^{-1}}, \quad J_{-o k}^{\text {sou }}=-\frac{1}{q} J_{i k}^{\text {sou }} .
$$

The phasors $J_{-c k}^{\text {ind }}, c=i, o ; k=1,2, \ldots, n$, are determined by the relation

$$
J_{c k}^{\text {ind }}=J_{c k}-J_{c k}^{\text {sou }},
$$

where $J_{c k}$ is the solution of the equation system (53).

Example 1. The inner conductor is of copper, and the outer conductor is of aluminium; the resistivity values at a temperature of $20^{\circ} \mathrm{C}$ have been taken over from [18]:

$$
\begin{gathered}
r_{i 0}=4 \mathrm{~mm}, \quad r_{\text {in }}=10 \mathrm{~mm}, \quad r_{\text {on }}=11 \mathrm{~mm}, \\
q=1.5, \quad \varrho_{i}=1.712 \times 10^{-8} \Omega \cdot \mathrm{m}, \\
\varrho_{o}=2.709 \times 10^{-8} \Omega \cdot \mathrm{m}, \\
U(t)=\sin \omega t, \quad \widehat{U}=1 \mathrm{~V} \cdot \mathrm{m}^{-1}, \quad \omega=2 \pi f .
\end{gathered}
$$

Figures 2 and 3 give (in continuous curves) the modules and arguments, respectively, of the phasors $J_{-c k}$ and $J_{-c k}^{\text {ind }}, c=$ $i, o ; k=1,2, \ldots, n$, in Example 1 for $f=10^{3} \mathrm{~Hz}$.

4.4. Inductance and Resistance of a Pair of Conductors. From the given voltage $\underline{U}$ and the calculated current phasor

$$
\underline{I}=a \sum_{k=1}^{n} \underline{J}_{i k}=-q a \sum_{k=1}^{n} \underline{J}_{o k},
$$

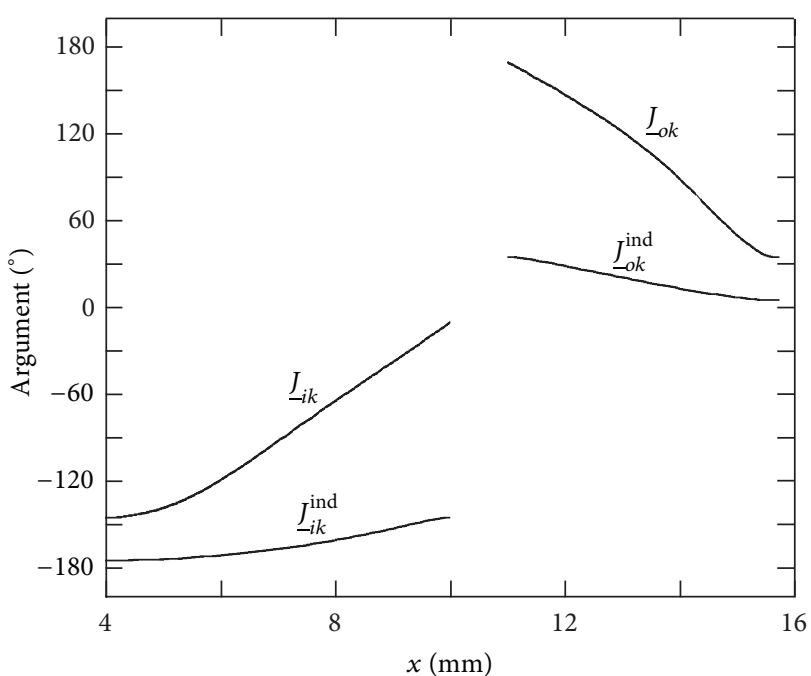

FIGURE 3: Dependence of phasor argument of resultant current density $J_{c k}$ and of phasor argument of induced current density $J_{-c k}^{\text {ind }}$ on distance from axis of conductors in Example 1, for $f=10^{3} \mathrm{~Hz}$, $n=1000$

inductance $L$ and resistance- $2 R_{s}$ of 1 metre of the considered pair of coaxial conductors can be determined. The considered segment of the two conductors can be replaced by the impedance

$$
\underline{Z}=\frac{\underline{U}}{\underline{I}}, \quad \underline{Z}=\Re(\underline{Z})+j \mathfrak{J}(\underline{Z})=R_{s}+j \omega L
$$

Using (60), $L$ and $R_{s}$ can be calculated for $\omega>0$. Admittedly, the value $L$ for $\omega=0$ has no meaning but it is equal to the limit of the values $L$ for $\omega \rightarrow 0+$ calculated using (60). For $\omega=0$, it holds that $R_{s}=R_{k} / n$. The values $R_{s}, L$ and the current phasor $\underline{I}=\widehat{I} \exp \left(j \beta_{c}\right)$ in the conductors together with the current density depend on $n$; see Tables 1 and 2 . The calculation of $R_{s}$ and $L$ corresponds with the case $n=1$ on the assumption that current density in the conductors is independent of the distance from the axis of conductors. This assumption evidently leads to the not very accurate values of $\widehat{I}$ and $L$.

Table 3 gives the dependence on $f$ of some quantities characterizing the pair of conductors in Example 1. The values $R_{s}$ and $L$ for $f=10^{-4} \mathrm{~Hz}$ in Table 3 can be regarded as the resistance- 2 and inductance values in the case $\omega=0$, as mentioned previously. The values given in Table 3 for $f>$ $10^{-4} \mathrm{~Hz}$ were obtained by calculating for $n=1000$. The current density amplitude in the inner conductor reaches its maximum $\widehat{J}_{\max }$ for $r=r_{i n}$.

The principle of calculating the resistance- 2 and inductance of the segment examined consists in replacing the segment of two conductors by impedance $\underline{Z}$, as given previously (see $(60))$. The examined segment of the two conductors is a parallel connection of $n$ partial loops, the equivalent 
TABLE 1: Dependence of current in inner conductor and impedance replacing $1 \mathrm{~m}$ of pair of conductors in Example 1 on $n$, for $f=50 \mathrm{~Hz}$.

\begin{tabular}{lcccccc}
\hline$n$ & 1 & 100 & 500 & 1000 & 2000 & 5000 \\
\hline$\widehat{I}(\mathrm{kA})$ & 7.19993 & 7.31824 & 7.31820 & 7.31820 & 7.31820 & 7.31819 \\
$\left.\beta_{i}{ }^{\circ}\right)$ & -16.295 & -11.216 & -11.231 & -11.233 & -11.234 & -11.234 \\
$R_{\mathrm{s}}\left(10^{-4} \Omega \cdot \mathrm{m}^{-1}\right)$ & 1.33311 & 1.34035 & 1.34029 & 1.34028 & 1.34028 \\
$L\left(10^{-8} \mathrm{H} \cdot \mathrm{m}^{-1}\right)$ & 12.4044 & 8.46042 & 8.47131 & 8.47266 & 8.47334 & 8.347374 \\
\hline
\end{tabular}

TABLE 2: Dependence of current in inner conductor and impedance replacing $1 \mathrm{~m}$ of pair of conductors in Example 1 on $n$, for $f=10^{6} \mathrm{~Hz}$.

\begin{tabular}{lcccccc}
\hline$n$ & 1 & 100 & 500 & 1000 & 2000 & 7000 \\
\hline$\widehat{I}(\mathrm{~A})$ & 1.28306 & 7.92151 & 7.78963 & 7.77231 & 7.76359 & 7.75834 \\
$\left.\beta_{i}{ }^{\circ}\right)$ & -89.990 & -86.070 & -86.040 & -86.047 & -86.051 & -86.054 \\
$R_{\mathrm{s}}\left(10^{-3} \Omega \cdot \mathrm{m}^{-1}\right)$ & 0.13331 & 8.65114 & 8.86500 & 8.86994 & 8.87057 & 8.87036 \\
$L\left(10^{-8} \mathrm{H} \cdot \mathrm{m}^{-1}\right)$ & 12.4044 & 2.00443 & 2.03829 & 2.04284 & 2.04515 & 2.04654 \\
\hline
\end{tabular}

impedances of which are $\underline{Z}_{k}, k=1,2, \ldots, n$, and therefore it holds that

$$
\frac{1}{\underline{Z}}=\sum_{k=1}^{n} \frac{1}{\underline{Z}_{k}}=a \sum_{k=1}^{n} \frac{\underline{J}_{i k}}{\underline{U}}=\frac{\underline{I}}{\underline{U}}
$$

The equivalent impedance $\underline{Z}_{k}$ of the segment of partial loops is illustrated in the Gaussian plane in Figure 4, for $f=10^{3} \mathrm{~Hz}$ in Example 1.

For $n=1$, the segment of the two conductors is identical to the segment of its only partial loop and it holds that $R_{s}=R_{k} / n$. This means that resistance- 2 of the segment under consideration does not depend on $f$, as confirmed by the data for $n=1$ in Tables 1 and 2 . In the case of $n=1, \mathfrak{R}(\underline{Z})$ can be regarded as resistance- 1 of the segment of both conductors. The situation is somewhat different for $n>1$.

The segments of partial loops satisfy equation system (53). These equations can be modified by breaking down the sum

$$
\begin{array}{r}
\left(\varrho_{i}+\frac{\varrho_{o}}{q}\right) J_{-i k}+j \omega \phi_{k k} J_{i k}+j \omega \sum_{\substack{\ell \neq k \\
\ell=1}}^{n} \phi_{k \ell} J_{i \ell}=\underline{U} \\
k=1,2 \ldots, n .
\end{array}
$$

Using the relation $\underline{I}_{k}=a J_{i k}$ yields

$$
R_{k} \underline{I}_{k}+j \omega L_{k} \underline{I}_{k}+j \omega \sum_{\substack{\ell \neq k \\ \ell=1}}^{n} M_{k \ell} \underline{I}_{\ell}=\underline{U}, \quad k=1,2 \ldots, n,
$$

where

$$
L_{k}=\frac{\phi_{k k}}{a}, \quad M_{k \ell}=\frac{\phi_{k \ell}}{a}, \quad \text { for } \ell \neq k .
$$

Dividing (63) by the current $\underline{I}_{k}$ leads to

$$
R_{k}+j \omega L_{k}+j \omega \underline{M}_{k}=\underline{Z}_{k}, \quad \underline{M}_{k}=\sum_{\substack{\ell \neq k \\ \ell=1}}^{n} M_{k \ell} \frac{\underline{I}_{\ell}}{\underline{I_{k}}} .
$$

The numbers $R_{k}, L_{k}, M_{k \ell}, \ell=1,2, \ldots k-1, k+1, \ldots, n$, are real numbers. $R_{k}$ is the resistance- 1 of 1 metre of the $k$ th partial loop. $L_{k}$ is the self-inductance of 1 metre of the $k$ th partial loop and $j \omega L_{k}$ is a pure imaginary number. $M_{k \ell}$ is the inductance of 1 metre of the $k$ th partial loop from the $\ell$ th partial loop. For $\ell \neq k, \omega>0$, the number $\underline{I}_{\ell} / \underline{I}_{k} \neq 1$ is complex, so that the third term on the left-hand side of the first equation in (65) is a complex number, whose parts are generally nonzero. It holds that

$$
\begin{gathered}
\mathfrak{R}\left(\underline{Z}_{k}\right)=R_{k}-\omega \mathfrak{I}\left(\underline{M}_{k}\right), \\
\mathfrak{J}\left(\underline{Z}_{k}\right)=\omega L_{k}+\omega \mathfrak{R}\left(\underline{M}_{k}\right) .
\end{gathered}
$$

The equaivalent impedance of the segment of the $k$ th partial loop can also be expressed by the sum

$$
\underline{Z}_{k}=R_{k}+\underline{Z}_{k}^{\text {ind }}
$$

where

$$
\underline{Z}_{k}^{\text {ind }}=j \omega\left(L_{k}+M_{k}\right)
$$

can be called induced impedance. The induced impedances $Z_{k}^{\text {ind }}$ are illustrated in the Gaussian plane in Figure 4 for $f=$ $10^{3} \mathrm{~Hz}$ in Example 1. Since some of the $\mathfrak{R}\left(\underline{Z}_{k}\right)$ values can be negative (see Figure 4), they cannot represent the resistance-1 of a real conductor.

4.5. Joule Power in Conductors. A current $a J_{i k}$ flows through the $k$ th partial loop, in which heat is released-Joule's dissipation power $P_{\mathrm{J}}\left(\right.$ in $\left.\mathrm{W} \cdot \mathrm{m}^{-1}\right)$. Let us write

$$
\begin{aligned}
P_{\mathrm{J}}(k) & =\frac{1}{2} \sum_{\ell=1}^{k}\left[\frac{\varrho_{i}}{a}\left(a \widehat{J}_{i \ell}\right)^{2}+\frac{\varrho_{o}}{q a}\left(q a \widehat{J}_{o \ell}\right)^{2}\right] \\
& =\frac{a}{2}\left(\varrho_{i}+\frac{\varrho_{o}}{q}\right) \sum_{\ell=1}^{k} \widehat{J}_{i \ell}^{2} .
\end{aligned}
$$

The value of $P_{\mathrm{J}}(k)$ calculated from relation (69) is equal to the Joule power in the considered segment of the first $k$ partial loops. The Joule power of the segment of both conductors under examination is thus $P_{\mathrm{J}}(n)$. 
TABle 3: Dependence of some quantities characterizing pair of conductors in Example 1 on $f$.

\begin{tabular}{|c|c|c|c|c|c|c|}
\hline $\begin{array}{l}f \\
\mathrm{~Hz}\end{array}$ & $\begin{array}{c}\widehat{J}_{\max } \\
\mathrm{kA} \cdot \mathrm{m}^{-2}\end{array}$ & $\begin{array}{l}\widehat{I} \\
\mathrm{~A}\end{array}$ & $\beta_{i}$ & $\begin{array}{c}R_{\mathrm{s}} \\
\mathrm{m} \Omega \cdot \mathrm{m}^{-1}\end{array}$ & $\begin{array}{c}L \\
10^{-8} \mathrm{H} \cdot \mathrm{m}^{-1}\end{array}$ & $\begin{array}{c}\omega L / R_{\mathrm{s}} \\
-\end{array}$ \\
\hline $10^{-4}$ & 28425.2 & 7501.24 & 0.00 & 0.13331 & 8.48427 & 0.00000 \\
\hline $10^{0}$ & 28425.2 & 7501.17 & -0.23 & 0.13331 & 8.48427 & 0.00400 \\
\hline $10^{1}$ & 28416.2 & 7493.64 & -2.29 & 0.13334 & 8.48381 & 0.03998 \\
\hline $10^{2}$ & 27665.1 & 6843.96 & -21.28 & 0.13616 & 8.43823 & 0.38940 \\
\hline $10^{3}$ & 23006.4 & 2067.35 & -55.12 & 0.27663 & 6.31529 & 1.43441 \\
\hline $10^{4}$ & 15705.7 & 442.434 & -66.99 & 0.88338 & 3.31113 & 2.35511 \\
\hline $10^{5}$ & 7467.02 & 66.5857 & -79.25 & 2.80166 & 2.34827 & 5.26638 \\
\hline $10^{6}$ & 2727.25 & 7.77231 & -86.05 & 8.86994 & 2.04284 & 14.4709 \\
\hline $10^{7}$ & 875.230 & 0.81751 & -88.69 & 27.9932 & 1.94631 & 43.6856 \\
\hline
\end{tabular}

Irrespective of the fact that $\mathfrak{R}\left(\underline{Z}_{k}\right)$ can have a negative value, the function $P_{\mathrm{Js}}(k)$ can be defined by a relation that is similar to relation (69):

$$
P_{\mathrm{Js}}(k)=\frac{1}{2} \sum_{\ell=1}^{k} \Re\left(\underline{Z}_{\ell}\right)\left(a \widehat{J}_{i \ell}\right)^{2}=\frac{a}{2} \sum_{\ell=1}^{k} \mathfrak{R}\left(\frac{\underline{U}}{\underline{J}_{i \ell}}\right) \widehat{J}_{i \ell}^{2}
$$

The values of the functions $P_{\mathrm{J}}(k)$ and $P_{\mathrm{Js}}(k)$ are shown in Figure 5 for the segment of a pair of conductors in Example 1 for $f=10^{3} \mathrm{~Hz}$. Let us add, for completeness, that not only the quantities $P_{\mathrm{J}}(k)$ and $P_{\mathrm{Js}}(k)$, but also $\mathfrak{R}\left(\underline{Z}_{k}\right)$ and $\mathfrak{\Im}\left(\underline{Z}_{k}\right)$ are functions of the natural number $k$ and thus acquire discrete values. In Figures 4 and 5, the discrete values are joined by continuous curves. The difference $P_{\mathrm{J}}(n)-P_{\mathrm{Js}}(n)$ is not nonzero but its magnitude converges to zero with increasing $n$. For $n=1000$, it holds that $P_{\mathrm{J}}(n)=591.50 \mathrm{~W} \cdot \mathrm{m}^{-1} P_{\mathrm{Js}}(n)=$ $591.35 \mathrm{~W} \cdot \mathrm{m}^{-1}$ in Example 1. Since relations (60) and (61) hold, $R_{\mathrm{s}}=\mathfrak{R}(\underline{Z})$ cannot be the resistance- 1 of the considered segment of conductors either, even if it holds that

$$
P_{\mathrm{Js}}(n) \doteq P_{\mathrm{Js}}(n)=\frac{1}{2} R_{\mathrm{s}} \widehat{I}^{2}
$$

4.6. Dependence of Inductance on $r_{i 0}$ and $f$. It follows from Table 3 that in a pair of conductors, the inductance, current in the conductors, and maximum magnitude of the current density depend on frequency $f$. The magnitude of current density in the inner conductor or outer conductor is an increasing or decreasing function, respectively, of the distance from the axis of both conductors, as follows from Figure 2 and from [16]. It is therefore relevant to deal with the dependence of $L$ on $r_{i 0}$ and $f$.

Example 2. The two coaxial conductors are of copper, having the same cross section $(q=1), r_{i n}=10 \mathrm{~mm}$, and insulation thickness $\theta=r_{\text {on }}-r_{\text {in }}=1 \mathrm{~mm}$.

Figure 6 gives the dependence of $L$ on $r_{i 0}$ and $f$ in Example $2(n=1000)$. For a given $r_{i n}$, the value

$$
L_{0}=\lim _{r_{i 0} \rightarrow r_{i n^{+}}} L
$$

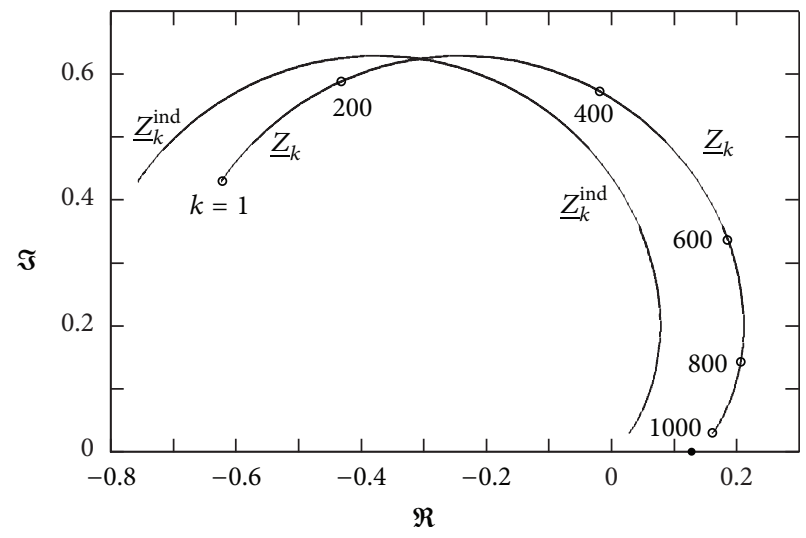

FIGURE 4: Equivalent and induced impedances $\underline{Z}_{k}$ and $\underline{Z}_{k}^{\text {ind }}$ of partial loops in Gaussian plane in Example 1, for $f=10^{3} \mathrm{~Hz}, n=1000$. Impedance parts are expressed in $\Omega \cdot \mathrm{m}^{-1}$. On horizontal axis, the value of resistance-1 of partial loop is marked by $\bullet$.

does not depend on $f$; however, $L_{0}$ depends on $\theta$. The following Table gives the values $L_{0}$ in Example 2:

$$
\begin{array}{ccccccc}
\theta(\mathrm{mm}) & 0 & 0.5 & 1 & 2 & 5 & 10 \\
\hline L_{0}\left(10^{-8} \mathrm{H} \cdot \mathrm{m}^{-1}\right) & 0 & 0.976 & 1.906 & 3.647 & 8.109 & 13.863
\end{array}
$$

\section{Comparison of Results}

No exact calculation of the impedance of a pair of coaxial tubular conductors has so far been made, leaving aside the so-called wire models [19]. The wire model replaces a solid conductor by cylindrical conductors of constant current density. In such a replacement, the conductor cross section is replaced by disjoint circles, which cannot fully cover the conductor cross section. The conductor cross section has a finite magnitude but when it is covered by circles, a finite part of the cross section still remains noncovered, even with the number of circles growing beyond all bounds. The quotient of the area of circles and the area of the cross section replaced by the circles can be $\approx 0.9$ as maximum.

Calculation methods are given in the following that are based on the assumption that the sought current density in 


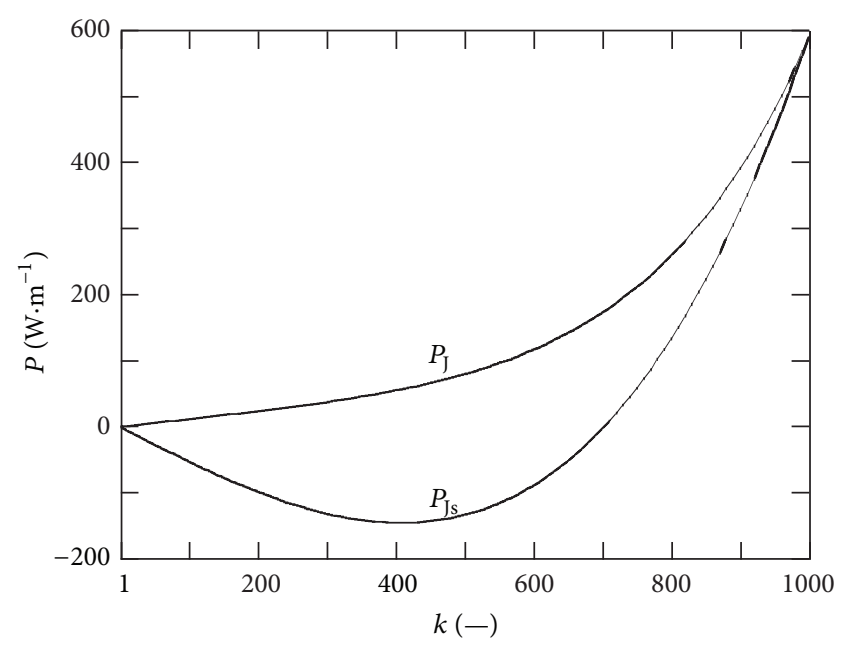

Figure 5: Dependence of $P_{\mathrm{J}}$ and $P_{\mathrm{Js}}$ values on $k$, for the segment of conductors in Example 1, for $f=10^{3} \mathrm{~Hz}, n=1000$.

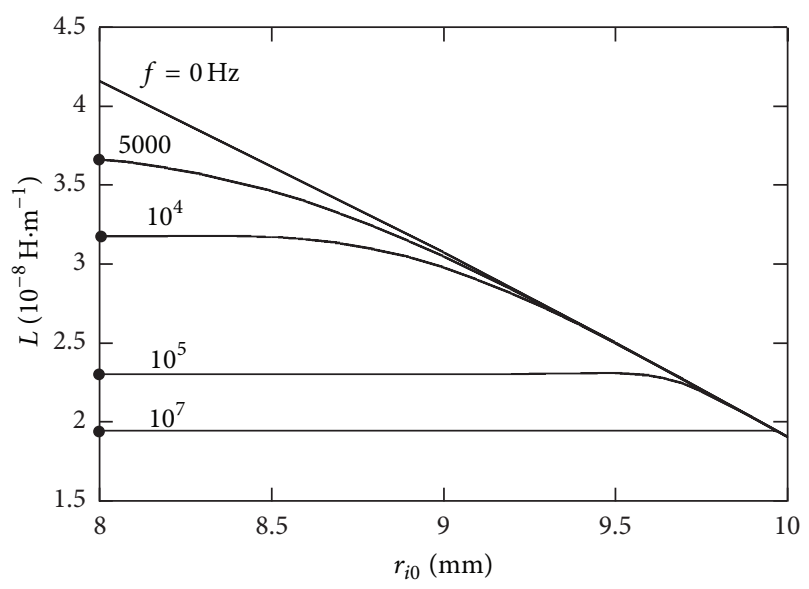

FIGURE 6: Dependence of inductance on radius $r_{i 0}$ and frequency $f$ in Example 2. Isolated points $\bullet$ denote values $L$ for $r_{i 0}=0 \mathrm{~mm}$. If $f=0$, then $L=9.226 \times 10^{-8} \mathrm{H} \cdot \mathrm{m}^{-1}$ for $r_{i 0}=0 \mathrm{~mm}$.

solid conductor is the solution of a second-order differential equation [20]. These methods are an analogy with the calculation of current density in tubular conductor [5, 17]. The boundary conditions necessary for the solution can be determined correctly only in the case that the inner conductor is cylindrical and the outer one is tubular with infinitely large radius $r_{o 0}[8,17,21]$; in the other cases, the boundary conditions are estimated [20] or the outer conductor is not considered.

The problematic solved in the present paper is to some extent related to the problematic of calculating induced current densities and earth impedances of overhead and buried conductors [22-24], in the solution of which, for example, the neural network artificial intelligence technique [25] can be employed.

Several formulae are given in the literature for an approximate calculation of the inductance of two coaxial solid conductors. It is true that these formulae can give a very exact value of inductance but only for some values of parameters (conductor dimensions and current frequency). However, the border between suitable and unsuitable parameters can only be determined via comparing approximate and exact inductance values.

The results of calculating $L$ using (60) will be compared with the values $L_{a}, a=\mathrm{H}, \mathrm{T}, \mathrm{M}, \mathrm{S}$, for a pair of coaxial copper conductors, $r_{i 0}=0 \mathrm{~mm}, r_{i n}=5 \mathrm{~mm}, q=1$, $\theta=0,1,2,3,4,5 \mathrm{~mm}, f=50,10^{3}, 10^{6} \mathrm{~Hz}$. The values of inductance $L(n=1000)$ and the percent deviations

$$
\Delta_{a}=100 \frac{L_{a}-L}{L}, \quad a=\mathrm{H}, \mathrm{T}, \mathrm{M}, \mathrm{S},
$$

are given in Table 4. The formula for calculating $L_{\mathrm{H}}$ is given in [26]. For the high frequencies $f \geq 10^{6} \mathrm{~Hz}$,

$$
L_{\mathrm{H}}=\frac{\mu_{0}}{2 \pi} \ln \frac{r_{o 0}}{r_{\mathrm{H}}}, \quad \text { where } r_{\mathrm{H}}= \begin{cases}r_{i n} & \text { for } r_{i 0}=0 \\ r_{i 0} & \text { for } r_{i 0}>0 .\end{cases}
$$

For the low frequencies $\left(f<10^{6} \mathrm{~Hz}\right.$ ), it is necessary to add 5 $\times 10^{-8} \mathrm{H} \cdot \mathrm{m}^{-1}$ on the right-hand side of (75), which according to [27] is the internal inductance of inner conductor. As can be seen from Table 4, formula (75) only gives orientational values of the inductance because when it was being derived, a constant current density in the cross section of conductors was assumed and part of the magnetic flux was neglected so that also the dependence on $f$ is vague. Formula (75) is acceptable for the low frequencies in the case that the size of conductor cross sections is small with respect to the size of the cross section of insulation between the conductors.

The formula for calculating $L_{\mathrm{T}}$ is given in [3]

$$
L_{\mathrm{T}}=\frac{\mu_{0}}{2 \pi} \ln \frac{r_{o 0}}{r_{i n}} .
$$

When deriving this formula, it was assumed that conductor thicknesses, that is, $r_{i n}-r_{i 0}$ and $r_{o 0}-r_{o n}$, were negligible with respect to $r_{i n}$ and $r_{o 0}$. This means that a current of finite magnitude only flows in an infinitely thin layer near the conductor surface. It is therefore no surprise that for a zero thickness of the insulation between conductors, $L_{\mathrm{T}}=0$, and the value of $L_{\mathrm{T}}$ is not very precise for a low $f$. Formula (76) has also been published in [28], with added assumption of $q=1$.

In [27], a formula is given for the calculation of inductance $L_{\mathrm{M}}$ "at sufficiently low frequencies"

$$
L_{\mathrm{M}}=L_{\mathrm{T}}+5 \times 10^{-8} \text {. }
$$

It is evident from Table 4 that for higher $f$ it would be more appropriate to put $L_{\mathrm{M}}=L_{\mathrm{T}}$ but a problem arises when determining the boundary between low and high frequency. Even if this boundary was established, the value of $L_{\mathrm{M}}$ exactly in the neighbourhood of this boundary would not be precise.

In [8], the formula for the calculation of inductance is derived on the assumption that $r_{i 0}=0, r_{\mathrm{o} 0}=\infty$, and $f>10^{5} \mathrm{~Hz}$ :

$$
L_{\mathrm{S}}=\frac{\mu_{0}}{2 \pi}\left[\ln \frac{r_{\text {on }}}{r_{\text {in }}}+\sqrt{\frac{\varrho_{i}}{2 \omega \mu_{0}}}\left(\frac{1}{r_{\text {in }}}+\frac{1}{r_{\text {on }}}\right)\right] .
$$


TABLE 4: Comparison of inductances for a pair of coaxial copper conductors, $r_{i 0}=0 \mathrm{~mm}, r_{i n}=5 \mathrm{~mm}, q=1$.

\begin{tabular}{|c|c|c|c|c|c|c|}
\hline$f(\mathrm{~Hz})$ & $\theta(\mathrm{mm})$ & $L\left(\mathrm{H} \cdot \mathrm{m}^{-1}\right)$ & $\Delta_{\mathrm{H}}(\%)$ & $\Delta_{\mathrm{T}}(\%)$ & $\Delta_{M}(\%)$ & $\Delta_{\mathrm{S}}(\%)$ \\
\hline \multirow{6}{*}{50} & 0 & $7.723 \times 10^{-8}$ & 54.5 & -100.0 & -35.3 & 382.4 \\
\hline & 1 & $1.064 \times 10^{-7}$ & 30.8 & -65.7 & -18.7 & 255.2 \\
\hline & 2 & $1.325 \times 10^{-7}$ & 19.7 & -49.2 & -11.5 & 191.8 \\
\hline & 3 & $1.559 \times 10^{-7}$ & 13.5 & -39.7 & -7.6 & 154.4 \\
\hline & 4 & $1.771 \times 10^{-7}$ & 9.8 & -33.6 & -5.4 & 130.0 \\
\hline & 5 & $1.965 \times 10^{-7}$ & 7.4 & -29.4 & -4.0 & 112.8 \\
\hline \multirow{6}{*}{$10^{3}$} & 0 & $6.913 \times 10^{-8}$ & 0.3 & -100.0 & -27.7 & 20.5 \\
\hline & 1 & $9.955 \times 10^{-8}$ & -10.4 & -63.4 & -13.1 & 13.3 \\
\hline & 2 & $1.264 \times 10^{-7}$ & -14.1 & -46.8 & -7.2 & 9.7 \\
\hline & 3 & $1.503 \times 10^{-7}$ & -15.5 & -37.5 & -4.2 & 7.5 \\
\hline & 4 & $1.719 \times 10^{-7}$ & -16.0 & -31.6 & -2.5 & 6.1 \\
\hline & 5 & $1.916 \times 10^{-7}$ & -16.0 & -27.6 & -1.5 & 5.0 \\
\hline \multirow{6}{*}{$10^{6}$} & 0 & $2.585 \times 10^{-9}$ & 2581.7 & -100.0 & 1834.4 & 1.9 \\
\hline & 1 & $3.885 \times 10^{-8}$ & 129.6 & -6.1 & 122.6 & 0.1 \\
\hline & 2 & $6.955 \times 10^{-8}$ & 56.0 & -3.2 & 68.7 & 0.0 \\
\hline & 3 & $9.616 \times 10^{-8}$ & 32.0 & -2.2 & 49.7 & 0.0 \\
\hline & 4 & $1.197 \times 10^{-7}$ & 20.7 & -1.8 & 40.0 & 0.0 \\
\hline & 5 & $1.407 \times 10^{-7}$ & 14.4 & -1.5 & 34.1 & 0.1 \\
\hline
\end{tabular}

A comparison of the inductance values in Table 4 reveals that Formula (78) is more accurate for the higher frequencies. The inductance $L_{S}$, calculated using (78), does not depend on $r_{\mathrm{i} 1}$ and $r_{\mathrm{o} 2}$, that is, no hindrance exactly at the higher frequencies. The unsuitability of (78) for the low frequencies follows from the fact that

$$
\lim _{\omega \rightarrow 0+} L_{S}=+\infty
$$

but, for example, for $\theta=2 \mathrm{~mm}$,

$$
\lim _{\omega \rightarrow 0+} L=1.325 \times 10^{-7} \mathrm{H} \cdot \mathrm{m}^{-1}
$$

and $L_{\mathrm{H}}=1.585 \times 10^{-7} \mathrm{H} \cdot \mathrm{m}^{-1}$. The reason is that inductance is defined independently of $f$ and must thus make sense also for $f=0$.

In the calculation of inductance, using (60), the method for calculating current density is of fundamental importance. One of the advantages of the latter method is that the current density can also be determined for $r_{\mathrm{i} 1}>0$. This advantage shows up in particular in the calculation of inductance at the lower frequencies, when the magnitude of current density is nonnegligible over the whole cross section of conductors.

\section{Conclusion}

The present paper is concerned with the definition and calculation of resistance $R$ and inductance $L$ of solid conductors. Attention is devoted, in particular, to the dependence of $L$ and $R$ on frequency $f$ under sinusoidal conditions. General considerations and conclusions are applied in the calculation of $L$ and $R$ of two infinitely long coaxial solid tubular conductors (coaxial conductors in the following). (i) A general formula (19) is derived for the calculation of conductance- 1 and thus also resistance- 1 of solid conductors.

(ii) Current filaments- 1 and filaments- 2 are defined. It is proved that a field $\mathbf{B}$ produced by filament- 1 is not defined on this filament. This knowledge is used to prove that the flux of vector $\mathbf{B}$ produced by a closed filament-1 which is in the shape of a curve $C$ through a surface bounded by the curve $C$ is not defined and thus the self-inductance of closed filament-1 cannot be calculated.

(iii) When deriving the method for the calculation of current density in coaxial conductors, it is assumed that the current density on the cross section of conductors is piecewise constant. When the conductors are supplied from a source of time-dependent voltage, the current density is the solution of a system of differential equations (52). If the voltage source is sinusoidal, then in the steady state, the current density is the solution of a system of algebraic equations (53) with complex coefficients. Using the calculated current density and formula (60), the $L$ and $R$ of coaxial conductors can be established.

(iv) Irrespective of the currently valid terminology and definitions [7], the still prevailing opinion is expressed by the following assertion: "the magnetic field of alternating current in a solid conductor displaces the current towards the conductor surface (skin effect) in consequence of which the resistance of the conductor increases." It follows from the analysis made that the current is not pushed but the current from the source is partially compensated by induced current. With increasing $f$, the resultant current 
flowing through the conductors decreases and their resistance- 2 increases while the resistance- 1 of the conductors does not change.

(v) A comparison is made of the exact value of the inductance of coaxial conductors established using formula (60), with the inductance values calculated using the approximate formulae (75)-(78) given in the literature.

\section{Acknowledgments}

This paper contains the results of research work funded until the end of 2011 from Research Plan no. MSM0021630516 of the Ministry of Education, Youth and Sports of the Czech Republic, and later from project of specific research program of Brno University of Technology no. FEKT-S-11-19.

\section{References}

[1] I. D. Mayergoyz and W. Lawson, Basic Electric Circuit Theory, Academic Press; Elsevier, New York, NY, USA, 1996.

[2] J. D. Jackson, Classical Electrodynamics, John Wiley \& Sons, New York, NY, USA, 2nd edition, 1975.

[3] I. E. Tamm, Fundamentals of the Theory of Electricity, Mir Publishers, Moscow, Russia, 1979.

[4] J. R. Reitz, F. J. Milford, and R. W. Christy, Foundations of Electromagnetic Theory, Addison-Wesley, New York, NY, USA, 1993.

[5] J. C. Maxwell, A Treatise on Electricity and Magnetism, vol. 1-2, Dover Publications, New York, NY, USA, 3d edition, 1954.

[6] O. Coufal, "On inductance and resistance of solitary long solid conductor," Acta Technica, vol. 57, no. 1, pp. 75-89, 2012.

[7] IEC, “60050-131:2002/A1:2008 International Electrotechnical Vocabulary-Part 131: Circuit theory".

[8] J. A. Stratton, Electromagnetic Theory, Adams Press, Chicago, Ill, USA, 2008.

[9] E. M. Purcell, Electricity and Magnetism, Berkeley Physics Course, vol. 2, Mc Graw-Hill, New York, NY, USA, 1984.

[10] O. D. Kellogg, Foundations of Potential Theory, Springer, Berlin, Germany, 1967.

[11] M. A. Rodríguez-Valverde and M. Tirado-Miranda, "A simpler derivation of the integralformula of electrical resistance," European Journal of Physics, vol. 30, pp. L47-L50, 2009.

[12] G. M. Fikhtengol'ts, Kurs Differentsial'nogo i Integral'nogo Ischisleniya, vol. 2, Publishing House FM, Moscow, Russia, 1962.

[13] K. Rektorys and E. Vitásek, Eds., Survey of Applicable Mathematics, Kluwer, Dordrecht, The Netherlands, 1994.

[14] IEC, “60050-121:1998 International Electrotechnical Vocabulary-Part 121: Electromagnetism".

[15] R. P. Feynman, R. B. Leighton, and M. Sands, The Feynman Lectures on Physics, the Definition Edition, vol. 3, AddisonWesley, Reading, Mass, USA, 2nd edition, 2005.

[16] O. Coufal, "Current density in a pair of solid coaxial conductors," Electromagnetics, vol. 27, no. 5, pp. 299-320, 2007.

[17] O. Coufal, "Current density in a long solitary tubular conductor," Journal of Physics A, vol. 41, no. 14, Article ID 145401, 2008.

[18] D. R. Lide, Handbook of Chemistry and Physics, CRC Press, Boca Raton, Fla, USA, 88th edition, 2008.
[19] A. Matsushima and H. Sakamoto, "Application of wire model to calculation of impedance of transmission lines with arbitrary cross sections," Electronics and Communications in Japan, Part II, vol. 85, no. 7, pp. 1-10, 2002.

[20] L. Yu. Mukoseev, Raspredelenie Peremennogo Toka v Tokoprovodakh, State Energetic Publishing House, Moscow, Russia, 1959.

[21] O. Coufal, "Comments on skin effect in solitary solid tubular conductor," Advances in Mathematical Physics, vol. 2011, Article ID 983678, 13 pages, 2011.

[22] D. D. Micu, G. Christoforidis, T. Papadopoulos, L. Czumbil, and D. Stet, "Accurate iterative algorithm to evaluate induced current densities and earth impedances of overhead and buried conductors in electromagnetic interference problems," in Proceedings of the 15th Biennal IEEE Conference on Electromagnetic Field Computation CEFC, Oita, Japan, November 2012.

[23] G. K. Papagiannis, D. A. Tsiamitros, D. P. Labridis, and P. S. Dokopoulos, "Direct numerical evaluation of earth return path impedances of underground cables," IEE Proceedings Generation Transmission and Distribution, vol. 152, no. 3, pp. 321-327, 2005.

[24] A. Ametani, T. Yoneda, Y. Baba, and N. Nagaoka, "An investigation of earth-return impedance between overhead and underground conductors and its approximation," IEEE Transactions on Electromagnetic Compatibility, vol. 51, no. 3, pp. 860-867, 2009.

[25] D. D. Micu, L. Czumbil, G. Christoforidis, and A. Ceclan, "Layer recurrent neural network solution for an electromagnetic interference problem," IEEE Transactions on Magnetics, vol. 47, no. 5, pp. 1410-1413, 2011.

[26] J. Hlávka, Ed., Electrical Engineering I, Physical Foundations, Part 1, SNTL, Prague, Czech Republic, 1968.

[27] R. E. Matick, Transmission Lines for Digital and Communication Networks, IEEE Press, New York, NY, USA, 1995.

[28] J. Bajcsy et al., Encyclopaedia of Electrical Engineering, vol. 1, (Theoretical Electrical Engineering), Alfa, Bratislava, Slovakia, 1977. 

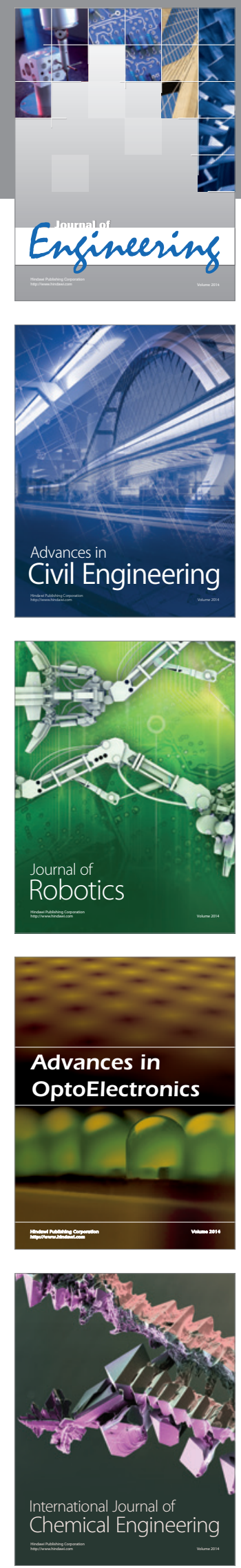

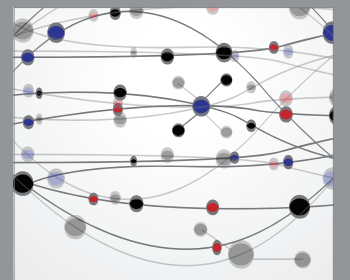

The Scientific World Journal
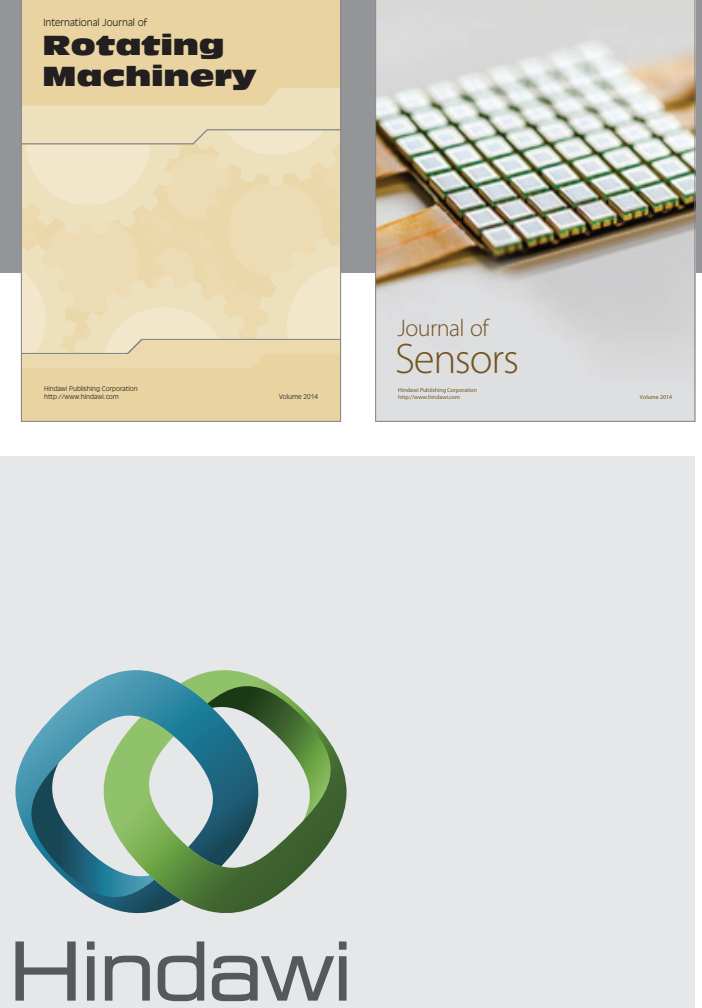

Submit your manuscripts at http://www.hindawi.com
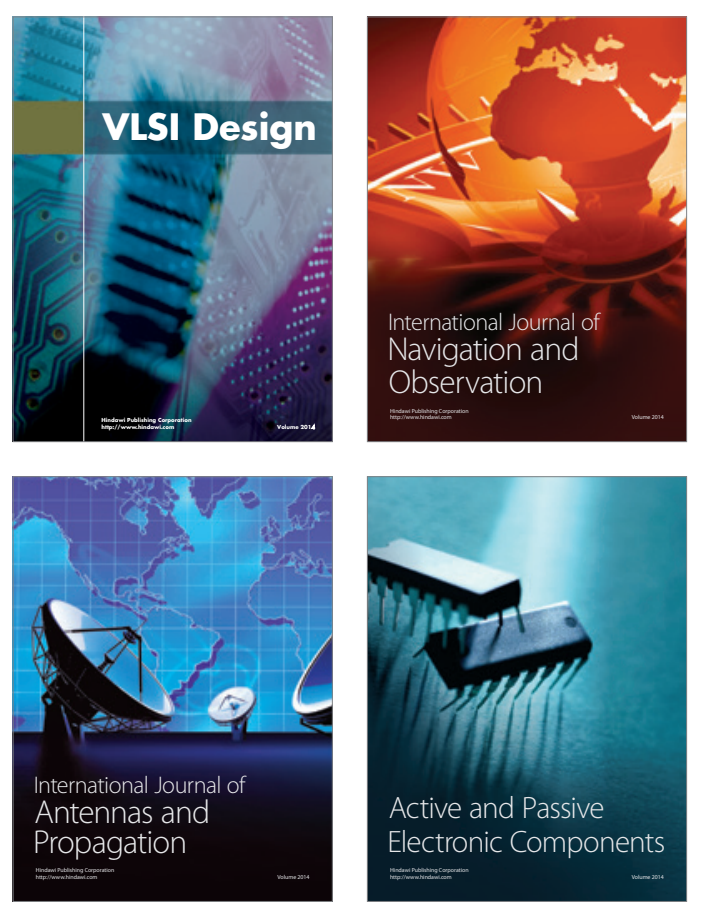
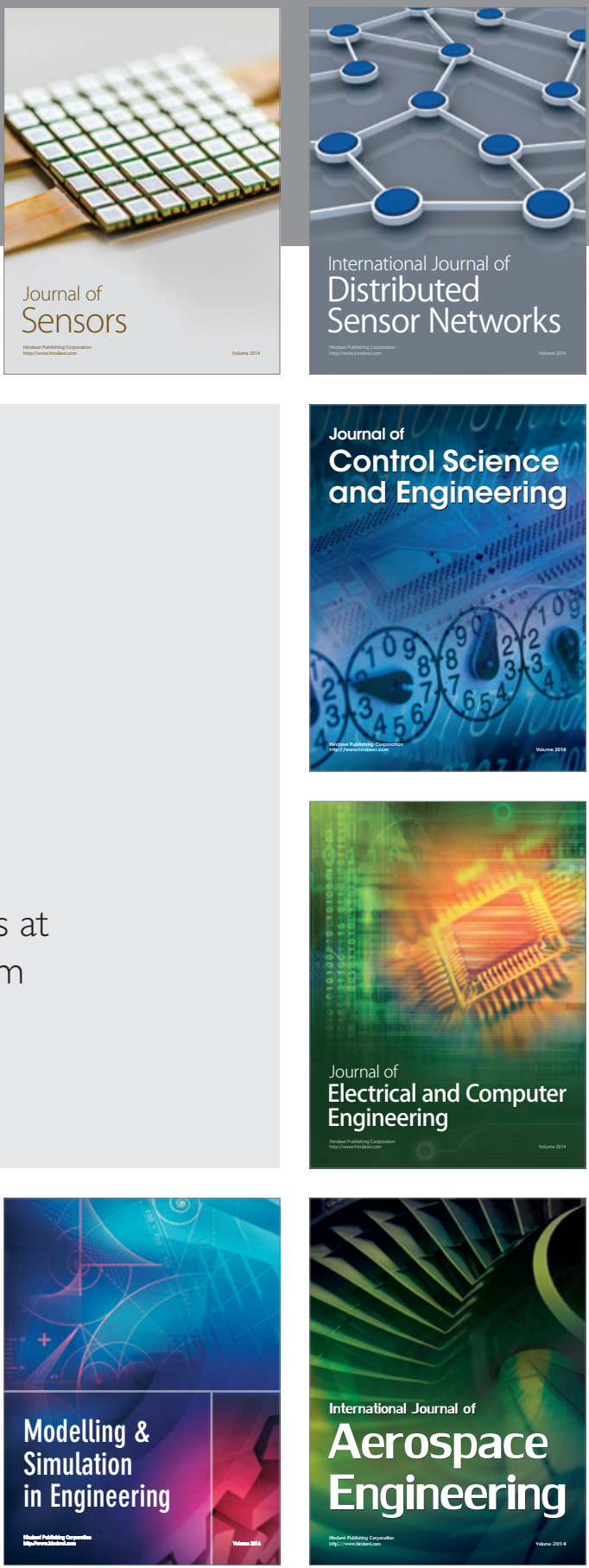

Journal of

Control Science

and Engineering
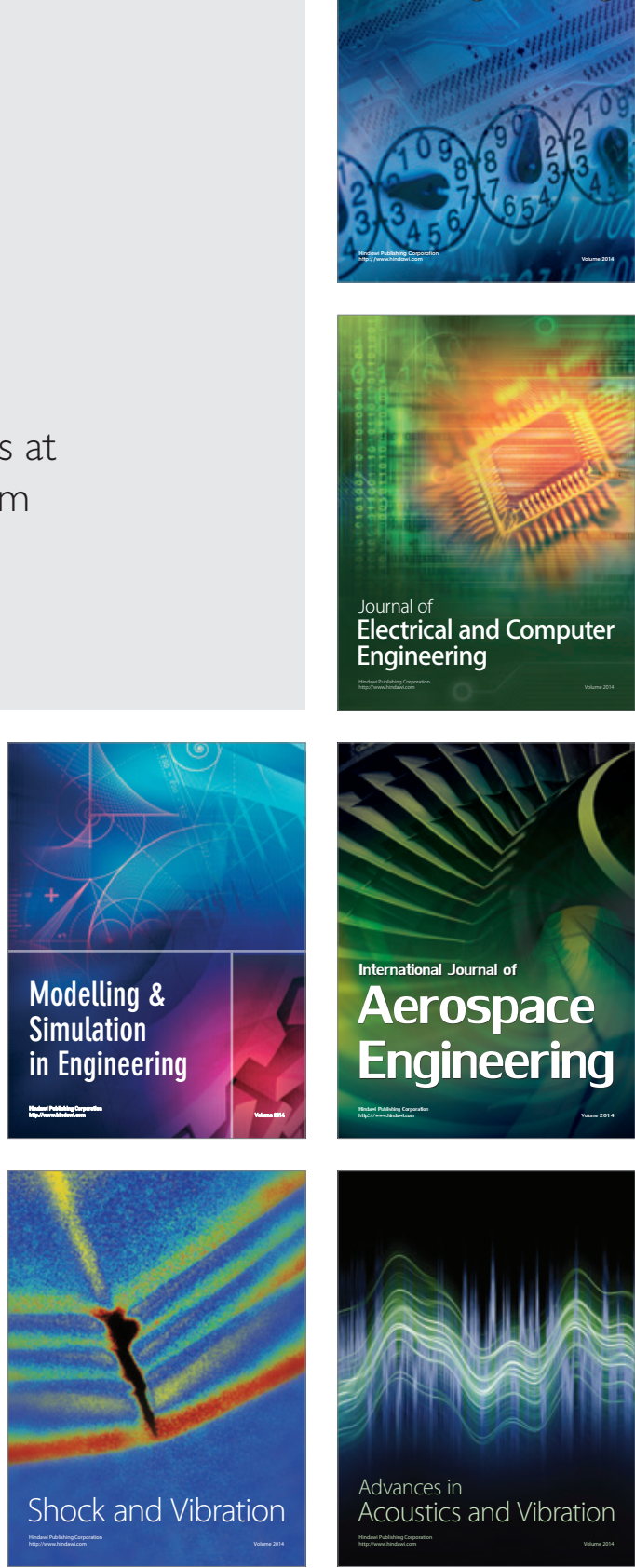